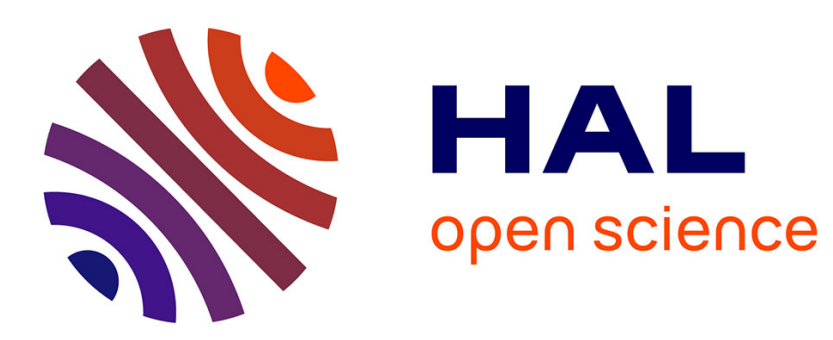

\title{
On the effective behavior of nonlinear inelastic composites: II A second-order procedure
}

\author{
Noël Lahellec, Pierre Suquet
}

\section{To cite this version:}

Noël Lahellec, Pierre Suquet. On the effective behavior of nonlinear inelastic composites: II A second-order procedure. Journal of the Mechanics and Physics of Solids, 2007, 55 (9), pp.1964-1992. 10.1016/j.jmps.2007.02.004 . hal-00214215

\section{HAL Id: hal-00214215 \\ https://hal.science/hal-00214215}

Submitted on 23 Jan 2008

HAL is a multi-disciplinary open access archive for the deposit and dissemination of scientific research documents, whether they are published or not. The documents may come from teaching and research institutions in France or abroad, or from public or private research centers.
L'archive ouverte pluridisciplinaire HAL, est destinée au dépôt et à la diffusion de documents scientifiques de niveau recherche, publiés ou non, émanant des établissements d'enseignement et de recherche français ou étrangers, des laboratoires publics ou privés. 


\title{
On the effective behavior of nonlinear inelastic composites: II. A second-order procedure.
}

\author{
Noël LAHELLEC ${ }^{a}$, Pierre SUQUET ${ }^{\mathrm{a}, *}$ \\ ${ }^{a}$ Laboratoire de Mécanique et d'Acoustique, CNRS and Aix-Marseille University, \\ 31 chemin Joseph Aiguier, 13402 Marseille cedex 20, France \\ E-mail: lahellec@lma.cnrs-mrs.fr,suquet@lma.cnrs-mrs.fr
}

\begin{abstract}
A new method for determining the overall behavior of composite materials comprised of nonlinear viscoelastic and elasto-viscoplastic constituents is presented. Part I of this work showed that upon use of an implicit time-discretization scheme, the evolution equations describing the constitutive behavior of the phases can be reduced to the minimization of an incremental energy function. This minimization problem is rigorously equivalent to a nonlinear thermoelastic problem with a transformation strain which is a nonuniform field (not even uniform within the phases). In part I of this paper the nonlinearity was handled using a variational (or secant) technique. In this second part of the study, a proper modification of the secondorder procedure of Ponte Castañeda is proposed and leads to replacing, at each time-step, the actual nonlinear viscoelastic composite by a linear viscoelastic one. The linearized problem is even further simplified by using an "effective internal variable" in each individual phase. The resulting predictions are in good agreement with exact results and improve on the predictions of the secant model proposed in part I of this paper.
\end{abstract}

Key words: Composites, homogenization, nonlinear viscoelasticity, elasto-viscoplasticity, variational method, second-order method, periodic micro-structure, random micro-structure, Hashin-Shtrikman estimates.

* Corresponding author 


\section{Introduction}

This article is the second part of a study devoted to the overall response of nonlinear composites comprised of phases which have a partly reversible and partly irreversible mechanical behavior (Lahellec and Suquet, 2006b). The individual constituents of the composites under consideration are generalized standard materials. As such, their mechanical state is characterized by their deformation $\varepsilon$ at a given material point and a collection of internal variables $\boldsymbol{\alpha}$ (Germain et al., 1983) and their constitutive relations derive from two thermodynamic potentials, a free-energy density $w$ and a dissipation potential $\varphi$. The driving forces available in the system to modify its state are obtained by derivation of the free-energy density with respect to the state variables :

$$
\boldsymbol{\sigma}=\frac{\partial w}{\partial \varepsilon}(\varepsilon, \boldsymbol{\alpha}), \quad \mathcal{A}=-\frac{\partial w}{\partial \boldsymbol{\alpha}}(\varepsilon, \boldsymbol{\alpha}) .
$$

The evolution of the internal variables is obtained by derivation of the dual of the dissipation potential $\varphi^{*}$ :

$$
\dot{\boldsymbol{\alpha}}=\frac{\partial \varphi^{*}}{\partial \mathcal{A}}(\mathcal{A})
$$

A representative volume element $V$ of a nonlinear composite comprised of $N$ different homogeneous phases, with free-energy $w^{(r)}$ and dissipation potential $\varphi^{(r)}$ is considered and subjected to a loading history. In part I of this study (Lahellec and Suquet, 2006b), an incremental variational principle has been derived following the lines of Mialon (1986), Ortiz and Stainier (1999) and Miehe (2002) (see part I of this study for more details). After time-discretization of the equations governing the quasi-static evolution of this representative volume element, it can be shown that the local fields of deformation and internal variables in the composite have a variational property which is used to reduce the problem of composites with two potentials to a problem for a nonlinear composite with a single potential. More specifically the overall stress $\boldsymbol{\Sigma}$ at time $t=t_{n+1}$ can be deduced from the strain $\boldsymbol{E}$ at time $t=t_{n+1}$ by derivation of a single condensed effective potential :

$$
\boldsymbol{\Sigma}=\frac{\partial \widetilde{w}_{\Delta}}{\partial \boldsymbol{E}}(\boldsymbol{E})
$$

where $\tilde{w}_{\Delta}$ has the variational characterization :

$$
\tilde{w}_{\Delta}(\boldsymbol{E})=\operatorname{Inf}_{\langle\boldsymbol{\varepsilon}\rangle=\boldsymbol{E}}\left\langle w_{\Delta}(\boldsymbol{\varepsilon})\right\rangle, \quad w_{\Delta}(\boldsymbol{\varepsilon})=\operatorname{Inf}_{\boldsymbol{\alpha}} J(\varepsilon, \boldsymbol{\alpha}),
$$

and

$$
J(\varepsilon, \boldsymbol{\alpha}, \boldsymbol{x})=\sum_{r=1}^{N}\left[w^{(r)}(\varepsilon, \boldsymbol{\alpha})+\Delta t \varphi^{(r)}\left(\frac{\boldsymbol{\alpha}-\boldsymbol{\alpha}_{n}(\boldsymbol{x})}{\Delta t}\right)\right] \chi^{(r)}(\boldsymbol{x}) .
$$


$w_{\Delta}$ is the local condensed potential, whereas $J$ is the incremental potential. For reasons exposed in Lahellec and Suquet (2006b), it is more convenient to work with $J$ rather than with $w_{\Delta}$. A first difference with the case of nonlinear composites with a single potential lies in the fact that the incremental potential $J$ not only depends on the strain $\varepsilon$ but also depends on the internal variables $\boldsymbol{\alpha}$. However, given the variational properties expressed by the relations (4), this additional dependence can be overcome by considering more variables in the minimization. Second, as noted in the first part of this study (Lahellec and Suquet, 2006b), two salient features of the incremental potential $J$ complicate the analysis and require suitable approximations :

a) The incremental potential $J$, just like the dissipation potential $\varphi^{(r)}$, is a nonquadratic function of $\boldsymbol{\alpha}$, even when the elasticity of the constituents is linear $\left(w^{(r)}\right.$ is quadratic). Therefore $J$ has to be approximated by a potential which is quadratic with respect to $\boldsymbol{\varepsilon}$ and $\boldsymbol{\alpha}$. This "linearization" procedure, which is usual in nonlinear composites with a single potential, amounts in the present setting to replacing the actual nonlinear viscoelastic (or elastoviscoplastic) composite by a linear viscoelastic one.

b) A second difficulty stemming from the dependence of $J$ on the field $\boldsymbol{\alpha}_{n}(\boldsymbol{x})$ requires a further approximation to approach $J$ by a piecewise uniform potential (as a function of $\boldsymbol{\varepsilon}$ and $\boldsymbol{\alpha}$ ). This is done, as in part I of this study, by introducing an effective internal variable (EIV) $\boldsymbol{\alpha}_{n}^{(r)}$ which replaces, as accurately as possible, the field $\boldsymbol{\alpha}_{n}(\boldsymbol{x})$ in phase $r$.

In the first part of this study, these two steps were taken care of simultaneously by a procedure derived from the variational procedure. But the two approximations are distinct in general and, in this part of the study, they will be handled in separate steps. The decoupling between the two approximations is illustrated by the case of linear viscoelasticity where no linearization is required but where the "effective internal variable" approximation is nevertheless needed (Lahellec and Suquet, 2006a).

Regarding the linearization strategy (point a), the procedure developed in part I of this study was based on the so-called variational method (in a form which has no real bounding property and which was therefore called a secant method). However, having in mind the improvements brought by second-order methods (Ponte Castañeda, 1996, 2002) over the variational method when the constitutive relations of the individual constituents are governed by a single potential, the use of one of these second-order estimates (SOE) is a natural alternative to the approach followed in the first part of the study. A first attempt in this direction is made in section 2, where a procedure based on the original second-order procedure is explored. However the resulting predictions are found to exhibit unrealistic features, such as a strong dependence of the predicted response on the time-step. After elucidating the origin of the problem, which lies in the gap between the overall stress field in the linear 
thermoelastic comparison composite and the derivative of the energy, use is made in section 3 of a modification due to Lahellec and Suquet (2004) of the original SOE. The resulting predictions are compared in section 5 with fullfield solutions and show no abnormal dependence on the time-step. They also improve significantly on the secant estimates derived in the first part of this study.

\section{A procedure based on the original second-order method}

\subsection{The original second-order procedure of Ponte Castañeda}

The idea of the original second-order method (SOE for second-order estimates) of Ponte Castañeda (1996) for nonlinear composites with a single potential is to replace the potential by its second-order Taylor expansion in the neighborhood of the average strain in each individual phase. In doing so, the initial nonlinear composite is replaced by a linear thermoelastic composite with anisotropic elastic moduli. The resulting prediction has no bounding property and yields only an estimate of the effective potential. On the other hand, this estimate is shown (Ponte Castañeda, 1996) to be exact to second-order in the contrast for nonlinear composites comprised of phases having a small contrast between their material properties (exact results for this case being available from the work of Suquet and Ponte Castañeda, 1993).

The SOE can be transposed in the present context of composites with two potentials (one of them, $w^{(r)}$ being quadratic), by replacing the nonquadratic potential $\varphi^{(r)}$ by its second-order Taylor expansion :

$$
\begin{aligned}
& \varphi^{(r)}\left(\frac{\boldsymbol{\alpha}-\boldsymbol{\alpha}_{n}}{\Delta t}\right) \simeq \varphi_{\text {soe }}^{(r)}\left(\frac{\boldsymbol{\alpha}-\boldsymbol{\alpha}_{n}}{\Delta t}\right) \\
& =\varphi^{(r)}\left(\dot{\boldsymbol{\alpha}}^{(r)}\right)+\frac{\partial \varphi^{(r)}}{\partial \dot{\boldsymbol{\alpha}}}\left(\dot{\boldsymbol{\alpha}}^{(r)}\right): \Delta \dot{\boldsymbol{\alpha}}+\frac{1}{2} \Delta \dot{\boldsymbol{\alpha}}: \frac{\partial^{2} \varphi^{(r)}}{\partial \dot{\boldsymbol{\alpha}}^{2}}\left(\dot{\boldsymbol{\alpha}}^{(r)}\right): \Delta \dot{\boldsymbol{\alpha}}
\end{aligned}
$$

where $\dot{\boldsymbol{\alpha}}^{(r)}$ is an effective rate for the internal variable in phase $r$ and

$$
\Delta \dot{\boldsymbol{\alpha}}=\frac{\boldsymbol{\alpha}-\boldsymbol{\alpha}_{n}}{\Delta t}-\dot{\boldsymbol{\alpha}}^{(r)}
$$

As initially proposed in Ponte Castañeda $(1996), \dot{\boldsymbol{\alpha}}^{(r)}$ is taken to be the phaseaverage of the rate of the internal variables :

$$
\dot{\boldsymbol{\alpha}}^{(r)}=\left\langle\frac{\boldsymbol{\alpha}-\boldsymbol{\alpha}_{n}}{\Delta t}\right\rangle_{r}=\langle\dot{\boldsymbol{\alpha}}\rangle_{r}
$$


Making the approximation (6) in the variational problem (4), amounts to replacing the incremental potential $J$ by :

$$
\begin{aligned}
J_{\text {soe }}(\varepsilon, \boldsymbol{\alpha}) & =\sum_{r=1}^{N}\left[w^{(r)}(\varepsilon, \boldsymbol{\alpha})+\Delta t \varphi_{\text {soe }}^{(r)}\left(\frac{\boldsymbol{\alpha}-\boldsymbol{\alpha}_{n}}{\Delta t}\right)\right] \chi^{(r)}(\boldsymbol{x}) \\
& =\sum_{r=1}^{N}\left[\frac{1}{2}(\varepsilon-\boldsymbol{\alpha}): \boldsymbol{L}^{(r)}:(\varepsilon-\boldsymbol{\alpha})+\right. \\
\Delta t\left(\varphi^{(r)}\left(\langle\dot{\boldsymbol{\alpha}}\rangle_{r}\right)\right. & \left.\left.+\frac{\partial \varphi^{(r)}}{\partial \dot{\boldsymbol{\alpha}}}\left(\langle\dot{\boldsymbol{\alpha}}\rangle_{r}\right): \Delta \dot{\boldsymbol{\alpha}}+\frac{1}{2} \Delta \dot{\boldsymbol{\alpha}}: \boldsymbol{L}_{v}^{(r)}: \Delta \dot{\boldsymbol{\alpha}}\right)\right] \chi^{(r)}(\boldsymbol{x}),
\end{aligned}
$$

where $\chi^{(r)}$ denotes the characteristic function of the domain $V_{r}$ occupied by the $r$-th phase and where :

$$
\boldsymbol{L}_{v}^{(r)}=\frac{\partial^{2} \varphi^{(r)}}{\partial \dot{\boldsymbol{\alpha}}^{2}}\left(\langle\dot{\boldsymbol{\alpha}}\rangle_{r}\right)
$$

Under this approximation, the variational problem (4) becomes :

$$
\widetilde{w}_{\Delta}(\boldsymbol{E}) \approx \widetilde{w}_{\Delta \text { soe }}(\boldsymbol{E})=\operatorname{Inf}_{\langle\boldsymbol{\varepsilon}\rangle=\boldsymbol{E}} \operatorname{Inf}_{\boldsymbol{\alpha}}\left\langle J_{\text {soe }}(\boldsymbol{\varepsilon}, \boldsymbol{\alpha})\right\rangle .
$$

The associated Euler-Lagrange equations read :

$$
\left\{\begin{array}{l}
\boldsymbol{\sigma}=\boldsymbol{L}^{(r)}:(\boldsymbol{\varepsilon}-\boldsymbol{\alpha})=\frac{\partial \varphi^{(r)}}{\partial \dot{\boldsymbol{\alpha}}}\left(\langle\dot{\boldsymbol{\alpha}}\rangle_{r}\right)+\boldsymbol{L}_{v}^{(r)}: \Delta \dot{\boldsymbol{\alpha}} \quad \text { in } V_{r} \\
\operatorname{div} \boldsymbol{\sigma}=0 \text { in } V,\langle\boldsymbol{\varepsilon}\rangle=\boldsymbol{E},+ \text { boundary conditions on } \partial V
\end{array}\right.
$$

The first line in (11) can be developed into

$\boldsymbol{\sigma}=\boldsymbol{L}^{(r)}:(\varepsilon-\boldsymbol{\alpha})=\boldsymbol{L}_{v}^{(r)}: \frac{\boldsymbol{\alpha}-\boldsymbol{\alpha}_{n}}{\Delta t}+\boldsymbol{\tau}^{(r)}, \quad \boldsymbol{\tau}^{(r)}=\frac{\partial \varphi^{(r)}}{\partial \dot{\boldsymbol{\alpha}}}\left(\langle\dot{\boldsymbol{\alpha}}\rangle_{r}\right)-\boldsymbol{L}_{v}^{(r)}: \dot{\boldsymbol{\alpha}}^{(r)}$

This last relation shows that the approximation (10) amounts to replacing the initial nonlinear composite by a linear thermo-viscoelastic composite with an anisotropic viscosity tensor $\boldsymbol{L}_{v}^{(r)}$, an initial (at time $t_{n}$ ) internal variable field $\boldsymbol{\alpha}_{n}(\boldsymbol{x})$ and a piecewise uniform eigenstress $\boldsymbol{\tau}^{(r)}$. Note that $\boldsymbol{\alpha}_{n}$ depends on $\boldsymbol{x}$ so that the thermo-viscoelastic problem (11) has to be solved numerically in general.

Once the equations (11) are solved for the local fields $\boldsymbol{\sigma}, \boldsymbol{\varepsilon}$ and $\boldsymbol{\alpha}$ the macroscopic stress-strain relations is obtained by derivation of the overall potential

$$
\Sigma_{\mathrm{soe}}=\frac{d \widetilde{w}_{\Delta \text { soe }}}{d \boldsymbol{E}}(\boldsymbol{E})
$$

In practice, the potential $\widetilde{w}_{\Delta \text { soe }}$ is not known explicitly as a function of $\boldsymbol{E}$ even when analytical schemes (such as the Hashin-Shtrikman bounds) are used to solve the thermoelastic problem (11). Therefore the derivative involved in (12) 
has to be computed numerically by taking difference quotients in $\boldsymbol{E}$. However, unexpected and inconsistent results, such as a significant dependence of the predictions on the time-step $\Delta t$ were obtained, as shown by the following example.

\subsection{An enlighting example}

The above comments are illustrated in figures 1 and 2 . We consider a periodic two-dimensional fiber-reinforced composite whose microstructure is characterized by the unit-cell shown in figure 6a. It is composed of an isotropic powerlaw nonlinear viscoelastic matrix reinforced by elastic fibers. The free-energy density in both phases is given by :

$$
\begin{gathered}
w^{(r)}(\varepsilon, \boldsymbol{\alpha})=\frac{1}{2}(\boldsymbol{\varepsilon}-\boldsymbol{\alpha}): \boldsymbol{L}^{(r)}:(\boldsymbol{\varepsilon}-\boldsymbol{\alpha}), \quad \boldsymbol{L}^{(r)}=3 k^{(r)} \boldsymbol{J}+2 \mu^{(r)} \boldsymbol{K} \\
k^{(r)}=\frac{E^{(r)}}{3\left(1-2 \nu^{(r)}\right)}, \quad \mu^{(r)}=\frac{E^{(r)}}{2\left(1+\nu^{(r)}\right)}
\end{gathered}
$$

where $\boldsymbol{J}$ and $\boldsymbol{K}$ are the two orthogonal projectors on spherical and deviatoric tensors respectively.

The dissipation potential is identically 0 in the fibers and reads in the matrix as :

$$
\varphi(\dot{\boldsymbol{\alpha}})=\frac{\sigma_{0} \dot{\varepsilon}_{0}}{m+1}\left(\frac{\dot{\alpha}_{\mathrm{eq}}}{\dot{\varepsilon}_{0}}\right)^{m+1},
$$

The material characteristics of the phases are :

- Fibers : $E=400 \mathrm{GPa}, \nu=0.2$

- Matrix : $E=70 \mathrm{GPa}, \nu=0.3, \sigma_{0}=480 \mathrm{MPa}, \dot{\varepsilon}_{0}=10^{-2} \mathrm{~s}^{-1}$ and $m=0.2$.

The fiber volume-fraction is $c^{(1)}=0.3$. The loading is an extension in the first direction :

$$
\boldsymbol{E}(t)=E_{11}(t) \boldsymbol{e}_{1} \otimes \boldsymbol{e}_{1}
$$

where $E_{11}$ is increased monotonically with constant strain-rate $E_{11}=510^{-3} t$.

The thermo(visco)elastic problem (11) is solved exactly by means of the Finite Element Method (FEM). Different time-steps have been used. The "exact" result is shown in figure 1 as a solid line, whereas the different predictions of the second-order procedure for different time-steps are shown by dashed lines. As can be seen, the second-order procedure shows a significant sensitivity of the predictions to the time-step. In particular, the asymptotic overall stress (as $t$ tends to $+\infty$ ) as predicted by the second-order procedure for different time-steps varies between to limits denoted as $\boldsymbol{\Sigma}_{\text {aff }}^{\infty}$ and $\boldsymbol{\Sigma}_{\text {soe }}^{\infty}$ (more details in section 2.4). It is close to $\Sigma_{\text {soe }}^{\infty}$ for large time-steps $(\Delta t=2 s)$ and close to $\Sigma_{\text {aff }}^{\infty}$ for small time-steps $\left(\Delta t=210^{-2} s\right)$. Another way of evaluating the 


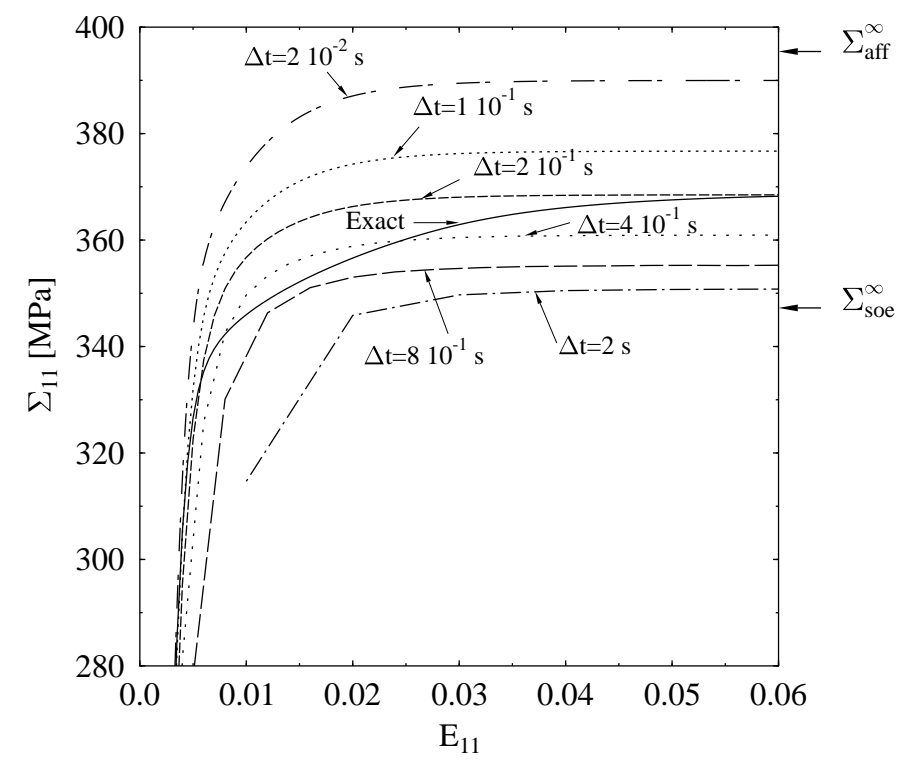

Fig. 1. Overall response of a fiber-reinforced composite under uniaxial extension at a constant strain-rate. Influence of the time-step $\Delta t$.

dependence of the predictions on the time-step is to consider the asymptotic predictions (at large times) of the stress obtained for different time-steps. This is done in figure 2 where the strong dependence of the asymptotic stress on the time-step can be seen.

\subsection{Second-order and affine procedures}

The original second-order method of Ponte Castañeda (1996), developed for composites governed by one nonquadratic potential, proceeds by approximating the initial potential by its Taylor expansion up to order 2. This expansion is a polynomial of degree 2 and the Euler-Lagrange equations associated with its minimization are those for a linear, anisotropic, thermoelastic composite. The effective energy of this thermoelastic composite is taken as an approximation for the actual effective potential and the overall stress is obtained by taking the derivative of this potential with respect to the overall strain as indicated by (12).

The affine method proposed by Masson and Zaoui (1999) (see also Masson et al., 2000, and Brenner and Masson, 2005, for subsequent developments) considers the Taylor expansion of the stress-strain relations in the phases up 


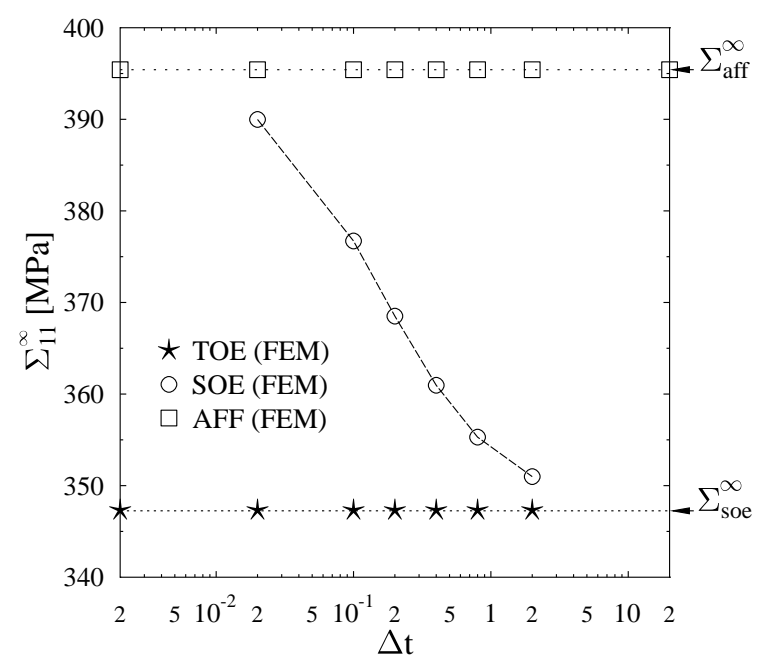

Fig. 2. Response of fiber-reinforced composites under monotone loading. Influence of the time-step $\Delta t$ on the asymptotic macroscopic stress $\Sigma_{11}^{\infty}=\lim _{t \rightarrow+\infty} \Sigma_{11}(t)$.

to order one (thus the method is called the affine method) and leads again to a linear thermoelastic problem, identical to that encountered in the secondorder procedure (expanding the potential to order 2 is equivalent to expanding the constitutive relations to order one). However there is a difference in the way in which the overall stress is deduced from the resolution of the thermoelastic problem. In the affine method, the overall stress is approximated by the average of the stress field in the thermoelastic composite and not by the derivative of the effective energy. The two results for the overall stress differ, although the affine and the second-order methods consider the same thermoelastic composite.

More specifically the affine prediction for the overall stress reads as :

$$
\Sigma_{\mathrm{aff}}=\langle\boldsymbol{\sigma}\rangle,
$$

which is different from (12) (Ponte Castañeda and Suquet, 1998). When applied to (11) the affine method showed none of the inconsistencies of the second-order method.

To understand the origin of the different sensitivity of the SOE and the affine methods, the difference between the two predictions can be explicitly evaluated. The overall stress predicted by the affine procedure reads as :

$$
\boldsymbol{\Sigma}_{\mathrm{aff}}=\sum_{r=1}^{N} c^{(r)} \boldsymbol{L}^{(r)}:\left(\langle\boldsymbol{\varepsilon}\rangle_{r}-\langle\boldsymbol{\alpha}\rangle_{r}\right)=\sum_{r=1}^{N} c^{(r)} \frac{\partial \varphi^{(r)}}{\partial \dot{\boldsymbol{\alpha}}}\left(\langle\dot{\boldsymbol{\alpha}}\rangle_{r}\right),
$$

whereas the stress $\boldsymbol{\Sigma}_{\text {soe }}$ is obtained by taking the derivative with respect to $\boldsymbol{E}$ 
of the homogenized incremental potential

$$
\begin{aligned}
& \widetilde{w}_{\Delta \text { soe }}(\boldsymbol{E})=\sum_{r=1}^{N} c^{(r)}\left\langle\frac{1}{2}(\varepsilon-\boldsymbol{\alpha}): \boldsymbol{L}^{(r)}:(\varepsilon-\boldsymbol{\alpha})\right. \\
& \left.+\Delta t\left[\varphi^{(r)}\left(\langle\dot{\boldsymbol{\alpha}}\rangle_{r}\right)+\frac{\partial \varphi^{(r)}}{\partial \dot{\boldsymbol{\alpha}}}\left(\langle\dot{\boldsymbol{\alpha}}\rangle_{r}\right): \Delta \dot{\boldsymbol{\alpha}}+\frac{1}{2} \Delta \dot{\boldsymbol{\alpha}}: \boldsymbol{L}_{v}^{(r)}: \Delta \dot{\boldsymbol{\alpha}}\right]\right\rangle_{r}
\end{aligned}
$$

Its derivative reads as :

$\frac{d \widetilde{w}_{\Delta \text { soe }}(\boldsymbol{E})}{d \boldsymbol{E}}=\sum_{r=1}^{N} c^{(r)}\left\langle(\boldsymbol{\varepsilon}-\boldsymbol{\alpha}): \boldsymbol{L}^{(r)}: \frac{d \boldsymbol{\varepsilon}}{d \boldsymbol{E}}+\Delta t\left[\frac{1}{2} \Delta \dot{\boldsymbol{\alpha}}: \frac{d \boldsymbol{L}_{v}^{(r)}}{d \dot{\boldsymbol{\alpha}}}: \frac{d\langle\dot{\boldsymbol{\alpha}}\rangle_{r}}{d \boldsymbol{E}}: \Delta \dot{\boldsymbol{\alpha}}\right]\right\rangle_{r}$

and finally

$$
\boldsymbol{\Sigma}_{\mathrm{soe}}=\boldsymbol{\Sigma}_{\mathrm{aff}}+\Delta t \sum_{r=1}^{N} c^{(r)}\left\langle\frac{1}{2} \Delta \dot{\boldsymbol{\alpha}}: \frac{d \boldsymbol{L}_{v}^{(r)}}{d \dot{\boldsymbol{\alpha}}}: \frac{d\langle\dot{\boldsymbol{\alpha}}\rangle_{r}}{d \boldsymbol{E}}: \Delta \dot{\boldsymbol{\alpha}}\right\rangle_{r} .
$$

\subsection{Why there should be no gap between the energy formulation and the affine procedure}

Consider the case where the loading history consists of an imposed average strain history with a constant (in time) strain-rate $\dot{\boldsymbol{E}}$. As already noticed in part I of this study (Lahellec and Suquet, 2006b), the overall response of the composite is governed initially (for small times $t \ll 1$ ) by its effective linear elastic properties (in other words by the effective potential $\tilde{w}$ alone) whereas its response at large times $(t \rightarrow+\infty)$ is governed by its viscous properties (in other words by the effective potential $\tilde{\varphi}$ alone). In the second limit the local fields $\boldsymbol{\sigma}$ and $\dot{\boldsymbol{\varepsilon}}$ tend to stationary values $\boldsymbol{\sigma}_{\infty}$ and $\dot{\boldsymbol{\varepsilon}}_{\infty}$ as $t$ tends to $+\infty$, the stress-rate $\dot{\boldsymbol{\sigma}}$ vanishing. It follows from the elastic relations that the difference $\dot{\boldsymbol{\varepsilon}}-\dot{\boldsymbol{\alpha}}$ tends to 0 and therefore that $\dot{\boldsymbol{\alpha}}$ can be asymptotically replaced by $\dot{\varepsilon}_{\infty}$ in the constitutive relations. The local problem solved by the local fields therefore reads asymptotically

$\boldsymbol{\sigma}_{\infty}=\frac{\partial \varphi^{(r)}}{\partial \dot{\boldsymbol{\varepsilon}}}\left(\dot{\boldsymbol{\varepsilon}}_{\infty}\right)$ in $V, \quad \operatorname{div} \boldsymbol{\sigma}_{\infty}=0$ in $V, \quad\left\langle\dot{\boldsymbol{\varepsilon}}_{\infty}\right\rangle=\dot{\boldsymbol{E}}+$ boundary conditions.

The standard SOE can now be applied to this problem with a single potential. The potential $\varphi^{r}$ in each phase is consequently replaced by its second-order Taylor expansion. This leads to approximating the fields $\boldsymbol{\sigma}_{\infty}$ and $\dot{\boldsymbol{\varepsilon}}_{\infty}$ by the solutions of a thermoelastic problem "tangent" to the nonlinear problem (20) (for simplicity, the corresponding local fields will still be denoted by $\boldsymbol{\sigma}_{\infty}$ and $\left.\dot{\boldsymbol{\varepsilon}}_{\infty}\right)$. Let $\boldsymbol{\Sigma}_{\text {aff }}^{\infty}$ denote the overall stress corresponding to the affine estimate for 
the problem (20):

$$
\boldsymbol{\Sigma}_{\mathrm{aff}}^{\infty}=\sum_{r=1}^{N} c^{(r)} \frac{\partial \varphi^{(r)}}{\partial \dot{\boldsymbol{\varepsilon}}}\left(\left\langle\dot{\boldsymbol{\varepsilon}}_{\infty}\right\rangle_{r}\right) .
$$

The affine estimate for the transient problem (finite $t$ ) obtained by the incremental procedure is given by (16) and is to be compared to (21). In the limit as $t$ tends to $+\infty$, one has $\dot{\boldsymbol{\varepsilon}}=\dot{\boldsymbol{\alpha}}$ and the estimates (16) and (21) coincide. The second-order estimate of the same problem (20), denoted by $\boldsymbol{\Sigma}_{\text {soe }}^{\infty}$, is given by (Ponte Castañeda and Suquet, 1998) :

$$
\boldsymbol{\Sigma}_{\mathrm{soe}}^{\infty}=\boldsymbol{\Sigma}_{\mathrm{aff}}^{\infty}+\sum_{r=1}^{N} c^{(r)}\left\langle\frac{1}{2}\left(\dot{\boldsymbol{\varepsilon}}_{\infty}-\left\langle\dot{\boldsymbol{\varepsilon}}_{\infty}\right\rangle_{r}\right): \frac{d \boldsymbol{L}_{v}^{(r)}}{d \dot{\boldsymbol{\varepsilon}}}: \frac{d\left\langle\dot{\boldsymbol{\varepsilon}}_{\infty}\right\rangle_{r}}{d \dot{\boldsymbol{E}}}:\left(\dot{\boldsymbol{\varepsilon}}_{\infty}-\left\langle\dot{\boldsymbol{\varepsilon}}_{\infty}\right\rangle_{r}\right)\right\rangle_{r},
$$

and is to be compared with (19). The two estimates $\boldsymbol{\Sigma}_{\text {aff }}$ and $\boldsymbol{\Sigma}_{\text {aff }}^{\infty}$ coincide asymptotically and it is sufficient to compare the last terms in (19) and (22). The following asymptotic results are worthy of notice :

$$
\lim _{t \rightarrow+\infty} \Delta \dot{\boldsymbol{\alpha}}=\dot{\boldsymbol{\varepsilon}}_{\infty}-\left\langle\dot{\boldsymbol{\varepsilon}}_{\infty}\right\rangle_{r} \quad \text { and } \quad \lim _{t \rightarrow+\infty} \frac{d \boldsymbol{L}_{v}^{(r)}}{d \dot{\boldsymbol{\alpha}}}\left(\langle\dot{\boldsymbol{\alpha}}\rangle_{r}\right)=\frac{d \boldsymbol{L}_{v}^{(r)}}{d \dot{\boldsymbol{\varepsilon}}}\left(\left\langle\dot{\boldsymbol{\varepsilon}}_{\infty}\right\rangle_{r}\right)
$$

It remains to compare the terms $\Delta t\left(d\langle\dot{\boldsymbol{\alpha}}\rangle_{r} / d \boldsymbol{E}\right)$ in $\boldsymbol{\Sigma}_{\text {soe }}$ and $d\left\langle\dot{\boldsymbol{\varepsilon}}_{\infty}\right\rangle_{r} / d \dot{\boldsymbol{E}}$ in $\Sigma_{\text {soe }}^{\infty}$. To this end, the relation (11a) can be averaged in phase $r$ :

$$
\boldsymbol{L}^{(r)}:\left(\langle\varepsilon\rangle_{r}-\langle\boldsymbol{\alpha}\rangle_{r}\right)=\frac{\partial \varphi^{(r)}}{\partial \dot{\boldsymbol{\alpha}}}\left(\langle\dot{\boldsymbol{\alpha}}\rangle_{r}\right)=\frac{\partial \varphi^{(r)}}{\partial \dot{\boldsymbol{\alpha}}}\left(\left\langle\frac{\boldsymbol{\alpha}-\boldsymbol{\alpha}_{n}}{\Delta t}\right\rangle_{r}\right),
$$

and this relation can be differentiated with respect to $\boldsymbol{E}$ to give :

$$
\frac{d\langle\boldsymbol{\alpha}\rangle_{r}}{d \boldsymbol{E}}=\left(\boldsymbol{L}^{(r)}+\frac{1}{\Delta t} \boldsymbol{L}_{v}^{(r)}\right)^{-1}: \boldsymbol{L}^{(r)}: \frac{d\langle\boldsymbol{\varepsilon}\rangle_{r}}{d \boldsymbol{E}},
$$

which implies, after time derivation :

$$
\Delta t \frac{d\langle\dot{\boldsymbol{\alpha}}\rangle_{r}}{d \boldsymbol{E}}=\left(\boldsymbol{L}^{(r)}+\frac{1}{\Delta t} \boldsymbol{L}_{v}^{(r)}\right)^{-1}: \boldsymbol{L}^{(r)}: \frac{d\langle\dot{\boldsymbol{\varepsilon}}\rangle_{r}}{d \dot{\boldsymbol{E}}}
$$

As a consequence :

$$
\lim _{t \rightarrow+\infty}\left[\Delta t \frac{d\langle\dot{\boldsymbol{\alpha}}\rangle_{r}}{d \boldsymbol{E}}\right]=\left(\boldsymbol{L}^{(r)}+\frac{1}{\Delta t} \boldsymbol{L}_{v}^{(r)}\right)^{-1}: \boldsymbol{L}^{(r)}: \frac{d\left\langle\dot{\boldsymbol{\varepsilon}}_{\infty}\right\rangle_{r}}{d \dot{\boldsymbol{E}}}
$$

This last relation sheds light on the problems encountered with the original second-order method:

- The second-order estimate (19) does not converge, as $t \rightarrow+\infty$, towards (22), as it should.

- The asymptotic limit of (19) depends on the elastic moduli of the phases (the $\boldsymbol{L}^{(r)}$ 's), on their dissipative properties (the $\boldsymbol{L}_{v}^{(r)}$ 's) and on the time-step $\Delta t$. This last point is illustrated in figure 2 . 
- It is only in the extreme case where $\boldsymbol{L}^{(r)} \gg \frac{1}{\Delta t} \boldsymbol{L}_{v}^{(r)}$ that the correct limit is retrieved.

- In the other extreme case $\boldsymbol{L}^{(r)} \ll \frac{1}{\Delta t} \boldsymbol{L}_{v}^{(r)}$, the second-order estimate (19) converges to the affine estimate $(21)$.

Remark 1 : Since the original second-order procedure of Ponte Castañeda (1996) which is followed here, other propositions have been made which lead to more accurate results in the context of composites governed by a single potential (see for instance Idiart et al., 2006a). In all these improved methods, the overall stress is computed as the derivative of the effective energy with respect to the overall strain and not as the average of the local stress field in the linear comparison composite (doing so would be in line with the above affine method). The gap between the two possible ways of evaluating the overall stress (derivative of the energy and average of the stress field in the linear comparison composite) is significantly reduced by these improved methods. However it is not clear that the dependence on the time-step would be removed by adopting one of these methods. Our guess is that the problem will persist as long as a gap between the two approaches, derivative of the energy and average of the stress field in the comparison composite, remains, but this statement deserves a deeper investigation which is left for future work.

\subsection{Orientation for the next section}

To avoid the above inconsistency in the prediction of the second-order method $\left(\lim _{t \rightarrow+\infty} \Sigma_{\text {soe }} \neq \Sigma_{\text {soe }}^{\infty}\right)$ it would be quite natural to make use of the affine method rather than of the second-order original approach. On the other hand the affine approach is known to be too stiff and less accurate than the secondorder procedure (for instance it is not exact to second-order in the contrast of the phases Masson et al., 2000). These limitations led us to develop a method having some of the features of the second-order method (based on energy considerations and exact to second-order in the contrast), combined with some of the features of the affine method (no dependence on the timestep). The direction that we have pursued is to enforce the stationarity of the effective potential $\tilde{w}_{\Delta}$ with respect to all (adjustable) variables defining the approach. This stationarity prescription, which was not present in the original second-order procedure of Ponte Castañeda (1996), ensures that the affine and energy-based approaches coincide

$$
\langle\boldsymbol{\sigma}\rangle=\frac{d \widetilde{w}_{\Delta}}{d \boldsymbol{E}}(\boldsymbol{E}),
$$

where $\boldsymbol{\sigma}$ is the stress field in the linear thermoelastic comparison composite. When this equality is satisfied, we can expect that there is no dependence on the time-step in such an approach (and the numerical results show that this 
guess is correct).

\section{A procedure based on a new second-order method}

\subsection{Reduction to a linearly viscoelastic problem}

Ponte Castañeda and Suquet (1998) have shown that, in the case of a single potential, the affine and the energy-based predictions differ by a term involving the third derivative of the potential. The relation (19) shows that in the present context they differ by a term involving the first derivative of $\boldsymbol{L}_{v}^{(r)}$ with respect to $\dot{\boldsymbol{\alpha}}$ or, equivalently, the third derivative of the dissipation potential $\varphi^{(r)}$.

Therefore, in order to close the gap between the affine and the second-order (or energy-based) method, the initial potential $\varphi^{(r)}$ is expanded to third-order and additional adjustable parameters are introduced in the third-order term in such a way that the resulting approximation remains a polynomial of degree 2. More specifically the initial potential $\varphi^{(r)}$ is replaced by the expression

$$
\varphi^{(r)}\left(\frac{\boldsymbol{\alpha}-\boldsymbol{\alpha}_{n}}{\Delta t}\right) \simeq \varphi_{\text {toe }}^{(r)}\left(\frac{\boldsymbol{\alpha}-\boldsymbol{\alpha}_{n}}{\Delta t}\right)
$$

where

$$
\begin{aligned}
& \varphi_{\text {toe }}^{(r)}\left(\frac{\boldsymbol{\alpha}-\boldsymbol{\alpha}_{n}}{\Delta t}\right)=\varphi^{(r)}\left(\dot{\boldsymbol{\alpha}}^{(r)}\right)+\frac{\partial \varphi^{(r)}}{\partial \dot{\boldsymbol{\alpha}}}\left(\dot{\boldsymbol{\alpha}}^{(r)}\right):\left(\frac{\boldsymbol{\alpha}-\boldsymbol{\alpha}_{n}}{\Delta t}-\dot{\boldsymbol{\alpha}}^{(r)}\right) \\
& +\frac{1}{2} \frac{\partial^{2} \varphi^{(r)}}{\partial \dot{\boldsymbol{\alpha}}^{2}}\left(\dot{\boldsymbol{\alpha}}^{(r)}\right)::\left(\frac{\boldsymbol{\alpha}-\boldsymbol{\alpha}_{n}}{\Delta t}-\dot{\boldsymbol{\alpha}}^{(r)}\right) \otimes\left(\frac{\boldsymbol{\alpha}-\boldsymbol{\alpha}_{n}}{\Delta t}-\dot{\boldsymbol{\alpha}}^{(r)}\right) \\
& +\frac{1}{6} \frac{\partial^{3} \varphi^{(r)}}{\partial \dot{\boldsymbol{\alpha}}^{3}}\left(\dot{\boldsymbol{\alpha}}^{(r)}\right)::\left(\frac{\boldsymbol{\alpha}-\boldsymbol{\alpha}_{n}}{\Delta t}-\dot{\boldsymbol{\alpha}}^{(r)}\right) \otimes\left(\widehat{\boldsymbol{\alpha}}^{(r)}-\dot{\boldsymbol{\alpha}}^{(r)}\right) \otimes\left(\widehat{\boldsymbol{\alpha}}^{(r)}-\dot{\boldsymbol{\alpha}}^{(r)}\right),
\end{aligned}
$$

where $\dot{\boldsymbol{\alpha}}^{(r)}$ (to be specified) is the uniform reference value for the rate of the internal variables in phase $r$ and where the last term, which looks like the third-order term in the Taylor expansion of the potential, except that it has been transformed into a first-order term by adding an additional adjustable variable $\widehat{\boldsymbol{\alpha}}^{(r)}$ which remains to be specified.

Substitution of the expansion (25) into the variational problem (4) leads to

$$
\widetilde{w}_{\Delta}(\boldsymbol{E})=\operatorname{Inf}_{\langle\boldsymbol{\varepsilon}\rangle=\boldsymbol{E}} \operatorname{Inf}_{\boldsymbol{\alpha}}\left\langle J_{\text {toe }}(\varepsilon, \boldsymbol{\alpha})\right\rangle,
$$

where $J_{\text {toe }}$ is defined as:

$$
J_{\text {toe }}(\varepsilon, \boldsymbol{\alpha})=\sum_{r=1}^{N}\left[w^{(r)}(\varepsilon, \boldsymbol{\alpha})+\Delta t \varphi_{\text {toe }}^{(r)}\left(\frac{\boldsymbol{\alpha}-\boldsymbol{\alpha}_{n}}{\Delta t}\right)\right] \chi^{(r)}(\boldsymbol{x}) .
$$


The variables $\dot{\boldsymbol{\alpha}}^{(r)}$ and $\widehat{\boldsymbol{\alpha}}^{(r)}$ are chosen to achieve stationarity in (26). In the proposed procedure, the final form of the variational problem (4) is :

$$
\widetilde{w}_{\Delta}(\boldsymbol{E})=\underset{\dot{\boldsymbol{\alpha}}^{(r)}}{\operatorname{Stat}_{\widehat{\boldsymbol{\alpha}}^{(r)}}} \operatorname{Stat}_{\langle\boldsymbol{\varepsilon}\rangle=\boldsymbol{E}} \operatorname{Inf}_{\boldsymbol{\alpha}}\left\langle J_{\text {toe }}(\varepsilon, \boldsymbol{\alpha})\right\rangle .
$$

The stationarity condition with respect to $\widehat{\boldsymbol{\alpha}}^{(r)}$ reads as:

$$
\left\langle\frac{\partial^{3} \varphi^{(r)}}{\partial \dot{\boldsymbol{\alpha}}^{3}}\left(\dot{\boldsymbol{\alpha}}^{(r)}\right):\left(\frac{\boldsymbol{\alpha}-\boldsymbol{\alpha}_{n}}{\Delta t}-\dot{\boldsymbol{\alpha}}^{(r)}\right) \otimes\left(\widehat{\boldsymbol{\alpha}}^{(r)}-\dot{\boldsymbol{\alpha}}^{(r)}\right)\right\rangle_{r}=0 .
$$

Several choices for $\dot{\boldsymbol{\alpha}}^{(r)}$ are possible in order to satisfy (28). The simplest one is to set $\dot{\boldsymbol{\alpha}}^{(r)}$ equal to the average of the time derivative of the internal variables in each individual phase :

$$
\dot{\boldsymbol{\alpha}}^{(r)}=\left\langle\frac{\boldsymbol{\alpha}-\boldsymbol{\alpha}_{n}}{\Delta t}\right\rangle_{r}=\langle\dot{\boldsymbol{\alpha}}\rangle_{r} .
$$

When this choice is made, the stationarity condition with respect to $\dot{\boldsymbol{\alpha}}^{(r)}$ reduces to :

$$
\frac{1}{3} \boldsymbol{N}_{v}^{(r)}\left(\langle\dot{\boldsymbol{\alpha}}\rangle_{r}\right)::\left(\widehat{\boldsymbol{\alpha}}^{(r)}-\langle\dot{\boldsymbol{\alpha}}\rangle_{r}\right) \otimes\left(\widehat{\boldsymbol{\alpha}}^{(r)}-\langle\dot{\boldsymbol{\alpha}}\rangle_{r}\right)=\boldsymbol{N}_{v}^{(r)}\left(\langle\dot{\boldsymbol{\alpha}}\rangle_{r}\right):: \boldsymbol{C}_{\dot{\boldsymbol{\alpha}}}^{(r)},
$$

with :

$$
\boldsymbol{N}_{v}^{(r)}\left(\langle\dot{\boldsymbol{\alpha}}\rangle_{r}\right)=\frac{\partial^{3} \varphi^{(r)}}{\partial \dot{\boldsymbol{\alpha}}^{3}}\left(\langle\dot{\boldsymbol{\alpha}}\rangle_{r}\right), \boldsymbol{C}_{\dot{\boldsymbol{\alpha}}}^{(r)}=\left\langle\left(\dot{\boldsymbol{\alpha}}-\langle\dot{\boldsymbol{\alpha}}\rangle_{r}\right) \otimes\left(\dot{\boldsymbol{\alpha}}-\langle\dot{\boldsymbol{\alpha}}\rangle_{r}\right)\right\rangle_{r}
$$

Again several choices for $\widehat{\boldsymbol{\alpha}}^{(r)}$ are possible in order to satisfy (30) depending on the specific form of the potentials $\varphi^{(r)}$. In the next section devoted to powerlaw materials, a specific choice is detailed for specific loading conditions.

Once the two stationarity conditions (28) and (30) are satisfied, the final expression for $J_{\text {toe }}(\boldsymbol{\varepsilon}, \boldsymbol{\alpha})$ is:

$$
\begin{aligned}
J_{\text {toe }}(\varepsilon, \boldsymbol{\alpha}) & =\sum_{r=1}^{N}\left[\frac{1}{2}(\varepsilon-\boldsymbol{\alpha}): \boldsymbol{L}^{(r)}:(\varepsilon-\boldsymbol{\alpha})+J_{r e f}^{(r)}\right. \\
+\boldsymbol{\tau}^{(r)} & \left.:\left(\boldsymbol{\alpha}-\boldsymbol{\alpha}_{n}\right)+\frac{1}{2}\left(\boldsymbol{\alpha}-\boldsymbol{\alpha}_{n}\right): \frac{1}{\Delta t} \boldsymbol{L}_{v}^{(r)}:\left(\boldsymbol{\alpha}-\boldsymbol{\alpha}_{n}\right)\right] \chi^{(r)}(\boldsymbol{x}),
\end{aligned}
$$

where

$$
\left.\begin{array}{rl}
\boldsymbol{L}_{v}^{(r)} & =\frac{\partial^{2} \varphi^{(r)}}{\partial \dot{\boldsymbol{\alpha}}^{2}}\left(\dot{\boldsymbol{\alpha}}^{(r)}\right), \\
J_{r e f}^{(r)} & =\Delta t\left[\varphi^{(r)}\left(\dot{\boldsymbol{\alpha}}^{(r)}\right)-\boldsymbol{\tau}^{(r)}: \dot{\boldsymbol{\alpha}}^{(r)}-\frac{1}{2} \dot{\boldsymbol{\alpha}}^{(r)}: \boldsymbol{L}_{v}^{(r)}: \dot{\boldsymbol{\alpha}}^{(r)}\right], \\
\boldsymbol{\tau}^{(r)} & =\frac{\partial \varphi^{(r)}}{\partial \dot{\boldsymbol{\alpha}}}\left(\dot{\boldsymbol{\alpha}}^{(r)}\right)-\boldsymbol{L}_{v}^{(r)}: \dot{\boldsymbol{\alpha}}^{(r)}+ \\
& \frac{1}{6} \boldsymbol{N}_{v}^{(r)}\left(\langle\dot{\boldsymbol{\alpha}}\rangle_{r}\right)::\left(\widehat{\boldsymbol{\alpha}}^{(r)}-\langle\dot{\boldsymbol{\alpha}}\rangle_{r}\right) \otimes\left(\widehat{\boldsymbol{\alpha}}^{(r)}-\langle\dot{\boldsymbol{\alpha}}\rangle_{r}\right) .
\end{array}\right\}
$$


The variational problem $(27)$ is then reduced to :

$$
\widetilde{w}_{\Delta}(\boldsymbol{E}) \simeq \operatorname{Inf}_{\langle\boldsymbol{\varepsilon}\rangle=\boldsymbol{E}} \operatorname{Inf}_{\boldsymbol{\alpha}}\left\langle J_{\text {toe }}(\varepsilon, \boldsymbol{\alpha})\right\rangle
$$

where $J_{\text {toe }}$ is given by (32). The associated local problem reads as :

$$
\left.\begin{array}{r}
\boldsymbol{\sigma}=\boldsymbol{L}^{(r)}:(\varepsilon-\boldsymbol{\alpha})=\boldsymbol{\tau}^{(r)}+\boldsymbol{L}_{v}^{(r)}:\left(\frac{\boldsymbol{\alpha}-\boldsymbol{\alpha}_{n}}{\Delta t}\right) \quad \text { in } V_{r}, \\
\operatorname{div} \boldsymbol{\sigma}=0 \text { in } V, \quad\langle\varepsilon\rangle=\boldsymbol{E}, \quad \text { periodicity conditions on } \partial V .
\end{array}\right\}
$$

One can recognize in (34) a problem for a generalized linear thermo-viscoelastic composite where the $r$-th individual phase is characterized by its elastic moduli $\boldsymbol{L}^{(r)}$, its viscous moduli $\boldsymbol{L}_{v}^{(r)}$, an eigenstress $\boldsymbol{\tau}^{(r)}$ and a field of internal variables $\boldsymbol{\alpha}_{n}$ at time $t_{n}$.

In conclusion, the linearization rule (25), in association with the stationarity conditions over $\dot{\boldsymbol{\alpha}}^{(r)}$ and $\widehat{\boldsymbol{\alpha}}^{(r)}$, has allowed us to reduce the minimization of the incremental potential to the minimization of a potential for an anisotropic linear viscoelastic problem. However, as in the case of one potential, the problem remains nonlinear because of the closing conditions (29) and (30). The coefficients of the linear viscoelastic composite depend on the solutions of these nonlinear equations which themselves depend on the local strain field in the linear thermo(visco)elastic composite.

Remark 2 : It is essential to note that, in this approach, the derivative of the effective energy $\widetilde{w}_{\Delta}$ with respect to the overall strain $\boldsymbol{E}$ coincides with the average of the stress in the linear thermo-viscoelastic composite. Indeed one has

$$
\frac{d \widetilde{w}_{\Delta}}{d \boldsymbol{E}}(\boldsymbol{E})=\left\langle\frac{\partial J_{\text {toe }}}{\partial \boldsymbol{\varepsilon}}: \frac{\partial \boldsymbol{\varepsilon}}{\partial \boldsymbol{E}}\right\rangle+\left\langle\frac{\partial J_{\text {toe }}}{\partial \boldsymbol{\alpha}}: \frac{\partial \boldsymbol{\alpha}}{\partial \boldsymbol{E}}+\frac{\partial J_{\text {toe }}}{\partial \dot{\boldsymbol{\alpha}}^{(r)}}: \frac{\partial \dot{\boldsymbol{\alpha}}^{(r)}}{\partial \boldsymbol{E}}+\frac{\partial J_{\text {toe }}}{\partial \hat{\boldsymbol{\alpha}}^{(r)}}: \frac{\partial \hat{\boldsymbol{\alpha}}^{(r)}}{\partial \boldsymbol{E}}\right\rangle .
$$

However, thanks to the stationarity conditions on $\boldsymbol{\alpha}, \dot{\boldsymbol{\alpha}}^{(r)}$ and $\widehat{\boldsymbol{\alpha}}^{(r)}$ in (27), the three last terms in these relations vanish. The first term can be transformed by Hill's lemma and the resulting relation reads as :

$$
\frac{d \widetilde{w}_{\Delta}}{d \boldsymbol{E}}(\boldsymbol{E})=\left\langle\frac{\partial J_{\text {toe }}}{\partial \boldsymbol{\varepsilon}}\right\rangle:\left\langle\frac{\partial \boldsymbol{\varepsilon}}{\partial \boldsymbol{E}}\right\rangle=\left\langle\frac{\partial J_{\text {toe }}}{\partial \boldsymbol{\varepsilon}}\right\rangle: \boldsymbol{I}=\langle\boldsymbol{\sigma}\rangle,
$$

where $\boldsymbol{\sigma}=\frac{\partial J_{\text {toe }}}{\partial \boldsymbol{\varepsilon}}(\boldsymbol{\varepsilon}, \boldsymbol{\alpha})$ is the stress field in the thermo-viscoelastic composite. 


\subsection{Resolution of the linear viscoelasticity problem}

The variational problem (26) with (32) involves the non piecewise uniform field of internal variables $\boldsymbol{\alpha}_{n}(\boldsymbol{x})$ arising from the previous time-step. This difficulty can be overcome by introducing an effective internal variable (EIV) $\boldsymbol{\alpha}_{n}^{(r)}$ at time $t_{n}$, following the same procedure as in part I of this study (Lahellec and Suquet, 2006b).

The potential $J_{\text {toe }}(\varepsilon, \boldsymbol{\alpha})$ is compared to a reference potential $J_{0}(\varepsilon, \boldsymbol{\alpha})$ given by :

$$
J_{0}(\varepsilon, \boldsymbol{\alpha})=\sum_{r=1}^{N} J_{0}^{(r)}(\varepsilon, \boldsymbol{\alpha}) \chi^{(r)}(\boldsymbol{x}),
$$

where

$J_{0}^{(r)}(\varepsilon, \boldsymbol{\alpha})=w^{(r)}(\varepsilon, \boldsymbol{\alpha})+J_{r e f}^{(r)}+\boldsymbol{\tau}^{(r)}:\left(\boldsymbol{\alpha}-\boldsymbol{\alpha}_{n}\right)+\frac{1}{2}\left(\boldsymbol{\alpha}-\boldsymbol{\alpha}_{n}^{(r)}\right): \frac{\theta^{(r)}}{\Delta t} \boldsymbol{L}_{v}^{(r)}:\left(\boldsymbol{\alpha}-\boldsymbol{\alpha}_{n}^{(r)}\right)$.

Note the two differences between $J_{0}$ and $J_{\text {toe }}$ given by (32). First, the field $\boldsymbol{\alpha}_{n}(\boldsymbol{x})$ of internal variables at time $t_{n}$ has been replaced in each individual constituent by an internal variable $\boldsymbol{\alpha}_{n}^{(r)}$ which is now uniform in phase $r$. Second, the viscous moduli $\boldsymbol{L}_{v}^{(r)}$ have been multiplied by a scalar factor $\theta^{(r)}$ which is closely linked to the replacement of the field $\boldsymbol{\alpha}_{n}(\boldsymbol{x})$ by $\boldsymbol{\alpha}_{n}^{(r)}$ as shown in Lahellec and Suquet (2006a) or Lahellec and Suquet (2006b) to which the reader is referred for more details. It should be noted that if $\theta^{(r)}$ is taken equal to 1 (no modification of the tangent moduli), then, by virtue of (40), this amounts to considering only fields $\boldsymbol{\alpha}_{n}(\boldsymbol{x})$ which have no fluctuations in the phases (piecewise uniform fields). As shown in the appendix A of the first part of this study (Lahellec and Suquet, 2006b), the resulting approach then reduces to the Transformation Field Analysis which is known to be too stiff.

With these two modifications in mind, $J_{\text {toe }}$ can be written as $J_{0}+\left(J_{\text {toe }}-J_{0}\right)$, which leads to replacing $\widetilde{w}_{\Delta}$ in (33) by :

$$
\widetilde{w}_{\Delta}(\boldsymbol{E})=\operatorname{Inf}_{\langle\boldsymbol{\varepsilon}\rangle=\boldsymbol{E}}\left[\operatorname{Inf}_{\boldsymbol{\alpha}}\left\langle J_{0}(\boldsymbol{\varepsilon}, \boldsymbol{\alpha})\right\rangle+\left\langle\operatorname{Stat}_{\boldsymbol{\alpha}}\left[J_{\text {toe }}(\varepsilon, \boldsymbol{\alpha})-J_{0}(\boldsymbol{\varepsilon}, \boldsymbol{\alpha})\right]\right\rangle\right] .
$$

The last stationarity problem, which is local in character, reads at each point $\boldsymbol{x}$ in phase $r$ :

$$
\operatorname{Stat}_{\boldsymbol{\alpha}} \frac{1}{2}\left(\boldsymbol{\alpha}-\boldsymbol{\alpha}_{n}\right): \frac{1}{\Delta t} \boldsymbol{L}_{v}^{(r)}:\left(\boldsymbol{\alpha}-\boldsymbol{\alpha}_{n}\right)-\frac{1}{2}\left(\boldsymbol{\alpha}-\boldsymbol{\alpha}_{n}^{(r)}\right): \frac{\theta^{(r)}}{\Delta t} \boldsymbol{L}_{v}^{(r)}:\left(\boldsymbol{\alpha}-\boldsymbol{\alpha}_{n}^{(r)}\right),
$$

whose solution is :

$$
\boldsymbol{\alpha}(\boldsymbol{x})=\sum_{r=1}^{N} \frac{\boldsymbol{\alpha}_{n}(\boldsymbol{x})-\theta^{(r)} \boldsymbol{\alpha}_{n}^{(r)}}{1-\theta^{(r)}} \chi^{(r)}(\boldsymbol{x})
$$


With this relation, the estimate (37) becomes:

$$
\operatorname{Inf}_{\langle\boldsymbol{\varepsilon}\rangle=\boldsymbol{E}}\left[\operatorname{Inf}_{\boldsymbol{\alpha}}\left\langle J_{0}(\boldsymbol{\varepsilon}, \boldsymbol{\alpha})\right\rangle\right]+\sum_{r=1}^{N} \frac{c^{(r)}}{2}\left\langle\left(\boldsymbol{\alpha}_{n}-\boldsymbol{\alpha}_{n}^{(r)}\right): \frac{\theta^{(r)}}{\Delta t\left(\theta^{(r)}-1\right)} \boldsymbol{L}_{v}^{(r)}:\left(\boldsymbol{\alpha}_{n}-\boldsymbol{\alpha}_{n}^{(r)}\right)\right\rangle_{r}
$$

This estimate can again be optimized with respect to $\theta^{(r)}$ and $\boldsymbol{\alpha}_{n}^{(r)}$ which have not yet been specified. Stationarity of (39) with respect to these variables yields

$$
\begin{gathered}
\frac{\partial}{\partial \theta^{(r)}}\left\langle J_{0}^{(r)}(\varepsilon, \boldsymbol{\alpha})+\frac{1}{2}\left(\boldsymbol{\alpha}_{n}-\boldsymbol{\alpha}_{n}^{(r)}\right): \frac{\theta^{(r)}}{\Delta t\left(\theta^{(r)}-1\right)} \boldsymbol{L}_{v}^{(r)}:\left(\boldsymbol{\alpha}_{n}-\boldsymbol{\alpha}_{n}^{(r)}\right)\right\rangle_{r}=0 \\
\Rightarrow \theta^{(r)}=1 \pm \sqrt{\frac{\left\langle\left(\boldsymbol{\alpha}_{n}-\boldsymbol{\alpha}_{n}^{(r)}\right): \boldsymbol{L}_{v}^{(r)}:\left(\boldsymbol{\alpha}_{n}-\boldsymbol{\alpha}_{n}^{(r)}\right)\right\rangle_{r}}{\left\langle\left(\boldsymbol{\alpha}-\boldsymbol{\alpha}_{n}^{(r)}\right): \boldsymbol{L}_{v}^{(r)}:\left(\boldsymbol{\alpha}-\boldsymbol{\alpha}_{n}^{(r)}\right)\right\rangle_{r}}}
\end{gathered}
$$

and

$$
\begin{gathered}
\frac{\partial}{\partial \boldsymbol{\alpha}_{n}^{(r)}}\left\langle J_{0}^{(r)}(\boldsymbol{\varepsilon}, \boldsymbol{\alpha})+\frac{1}{2}\left(\boldsymbol{\alpha}_{n}-\boldsymbol{\alpha}_{n}^{(r)}\right): \frac{\theta^{(r)}}{\Delta t\left(\theta^{(r)}-1\right)} \boldsymbol{L}_{v}^{(r)}:\left(\boldsymbol{\alpha}_{n}-\boldsymbol{\alpha}_{n}^{(r)}\right)\right\rangle_{r}=0 \\
\Rightarrow \quad \boldsymbol{\alpha}_{n}^{(r)}=\frac{\left\langle\boldsymbol{\alpha}_{n}\right\rangle_{r}+\left(\theta^{(r)}-1\right)\langle\boldsymbol{\alpha}\rangle_{r}}{\theta^{(r)}}
\end{gathered}
$$

The expression (39) can be further simplified by eliminating $\boldsymbol{\alpha}$. The field $\boldsymbol{\alpha}$ solution of the infimum problem in (39) is given in phase $r$ by :

$$
\begin{aligned}
\boldsymbol{\alpha}(\boldsymbol{x})= & \left(\boldsymbol{L}^{(r)}+\frac{\theta^{(r)}}{\Delta t} \boldsymbol{L}_{v}^{(r)}\right)^{-1}: \boldsymbol{L}^{(r)}: \boldsymbol{\varepsilon}(\boldsymbol{x}) \\
& +\left(\boldsymbol{L}^{(r)}+\frac{\theta^{(r)}}{\Delta t} \boldsymbol{L}_{v}^{(r)}\right)^{-1}:\left(\frac{\theta^{(r)}}{\Delta t} \boldsymbol{L}_{v}^{(r)}: \boldsymbol{\alpha}_{n}^{(r)}-\boldsymbol{\tau}^{(r)}\right)
\end{aligned}
$$

Upon substitution of this expression in (39), the condensed effective potential can be estimated as:

$$
\widetilde{w}_{0}(\boldsymbol{E})+\sum_{r=1}^{N} \frac{c^{(r)}}{2}\left\langle\left(\boldsymbol{\alpha}_{n}-\boldsymbol{\alpha}_{n}^{(r)}\right): \frac{\theta^{(r)}}{\Delta t\left(\theta^{(r)}-1\right)} \boldsymbol{L}_{v}^{(r)}:\left(\boldsymbol{\alpha}_{n}-\boldsymbol{\alpha}_{n}^{(r)}\right)\right\rangle_{r} .
$$

In this expression $\tilde{w}_{0}$ is the effective energy of an auxiliary thermoelastic problem :

$$
\tilde{w}_{0}(\boldsymbol{E})=\operatorname{Inf}_{\langle\boldsymbol{\varepsilon}\rangle=\boldsymbol{E}}\left\langle w_{0}(\boldsymbol{\varepsilon})\right\rangle,
$$

with

$$
w_{0}(\boldsymbol{\varepsilon})=\sum_{r=1}^{N} w_{0}^{(r)}(\boldsymbol{\varepsilon}) \chi^{(r)}(\boldsymbol{x}), \quad w_{0}^{(r)}(\boldsymbol{\varepsilon})=\frac{1}{2} \boldsymbol{\varepsilon}: \mathcal{L}_{0}^{(r)}: \boldsymbol{\varepsilon}+\boldsymbol{\rho}_{0}^{(r)}: \boldsymbol{\varepsilon}+f_{0}^{(r)},
$$


where the tensors $\mathcal{L}_{0}^{(r)}$ and $\boldsymbol{\rho}_{0}^{(r)}$ and the energy $f_{0}^{(r)}$ are piecewise uniform and defined as :

$$
\left.\begin{array}{l}
\mathcal{L}_{0}^{(r)}=\boldsymbol{L}^{(r)}:\left(\boldsymbol{L}^{(r)}+\frac{\theta^{(r)}}{\Delta t} \boldsymbol{L}_{v}^{(r)}\right)^{-1}: \frac{\theta^{(r)}}{\Delta t} \boldsymbol{L}_{v}^{(r)} \\
\boldsymbol{\rho}_{0}^{(r)}=\boldsymbol{L}^{(r)}:\left(\boldsymbol{L}^{(r)}+\frac{\theta^{(r)}}{\Delta t} \boldsymbol{L}_{v}^{(r)}\right)^{-1}:\left[\boldsymbol{\tau}^{(r)}-\frac{\theta^{(r)}}{\Delta t} \boldsymbol{L}_{v}^{(r)}: \boldsymbol{\alpha}_{n}^{(r)}\right] \\
f_{0}^{(r)}=J_{r e f}^{(r)}-\boldsymbol{\tau}^{(r)}:\left\langle\boldsymbol{\alpha}_{n}\right\rangle_{r}+\frac{1}{2} \boldsymbol{\alpha}_{n}^{(r)}: \frac{\theta^{(r)}}{\Delta t} \boldsymbol{L}_{v}^{(r)}: \boldsymbol{\alpha}_{n}^{(r)} \\
-\frac{1}{2}\left(\boldsymbol{\tau}^{(r)}+\frac{\theta^{(r)}}{\Delta t} \boldsymbol{L}_{v}^{(r)}: \boldsymbol{\alpha}_{n}^{(r)}\right):\left(\boldsymbol{L}^{(r)}+\frac{\theta^{(r)}}{\Delta t} \boldsymbol{L}_{v}^{(r)}\right)^{-1}:\left(\boldsymbol{\tau}^{(r)}+\frac{\theta^{(r)}}{\Delta t} \boldsymbol{L}_{v}^{(r)}: \boldsymbol{\alpha}_{n}^{(r)}\right)
\end{array}\right\}
$$

The first and second-order moments of the strain field (in the thermoelastic body) are given by (see for instance appendix C of part I of this study) :

$$
\langle\boldsymbol{\varepsilon}\rangle_{r}=\boldsymbol{A}_{0}^{(r)}: \boldsymbol{E}+\boldsymbol{a}_{0}^{(r)} \text { and }\langle\boldsymbol{\varepsilon} \otimes \boldsymbol{\varepsilon}\rangle_{r}=\frac{2}{c^{(r)}} \frac{\partial \tilde{w}_{0}}{\partial \mathcal{L}_{0}^{(r)}}
$$

where $\boldsymbol{A}_{0}^{(r)}, \boldsymbol{a}_{0}^{(r)}$ are the classical localization tensors in a thermoelastic composite with the same microstructure as the actual nonlinear composite. They can be obtained either numerically or analytically for specific microstructures.

Remark 3 : Using the same arguments (stationarity conditions) as in remark 2 , one can prove that

$$
\frac{d \widetilde{w}_{\Delta}}{d \boldsymbol{E}}(\boldsymbol{E})=\left\langle\frac{\partial J_{0}}{\partial \boldsymbol{\varepsilon}}(\varepsilon, \boldsymbol{\alpha})\right\rangle=\langle\boldsymbol{\sigma}\rangle
$$

where $\boldsymbol{\sigma}$ is now the stress field in the thermoelastic composite defined by the relations (45).

Remark 4 : As in the first part of this study, two different expressions (38) and (42) for the field of internal variables $\boldsymbol{\alpha}$ have been derived. Let $\boldsymbol{\alpha}^{*}$ and $\boldsymbol{\alpha}^{* *}$ denote respectively these two different expressions. The first one solves the stationarity condition in the evaluation of the gap between $J$ and $J_{0}$ whereas the second realizes the infimum condition which serves to define the thermoelastic potential $w_{0}$ from the potential $J_{0}$.

A straightforward calculation starting from (37) shows that the stationarity conditions (40) and (41) express equality between the first moment of $\boldsymbol{\alpha}^{*}$ and $\boldsymbol{\alpha}^{* *}$ in each individual phase and equality of a scalar quantity related to the second moment of these fields :

$$
\langle\boldsymbol{\alpha}\rangle_{r}=\left\langle\boldsymbol{\alpha}^{*}\right\rangle_{r} \text { and } \boldsymbol{L}_{v}^{(r)}::\langle\boldsymbol{\alpha} \otimes \boldsymbol{\alpha}\rangle_{r}=\boldsymbol{L}_{v}^{(r)}::\left\langle\boldsymbol{\alpha}^{*} \otimes \boldsymbol{\alpha}^{*}\right\rangle_{r}
$$




\subsection{Effective response of the composite}

Let us first note that four unknowns are a priori to be determined, three of them being tensorial $\dot{\boldsymbol{\alpha}}^{(r)}, \boldsymbol{\alpha}_{n}^{(r)}, \widehat{\boldsymbol{\alpha}}^{(r)}$ and one unknown being scalar, $\theta^{(r)}$. However, the relations (29) and (41) show that these unknowns are related in such a way that one of them can be eliminated. It was chosen to work with $\langle\boldsymbol{\alpha}\rangle_{r}, \widehat{\boldsymbol{\alpha}}^{(r)}$ and $\theta^{(r)}$ as the main unknowns.

The procedure for determining the effective response of the composite then goes as follows:

1. At time $t_{n}$, the first moment $\left\langle\boldsymbol{\alpha}_{n}\right\rangle_{r}$ and the second moment $\left\langle\boldsymbol{\alpha}_{n}: \boldsymbol{\alpha}_{n}\right\rangle_{r}$ of the internal variables are known in each individual phase $r$.

2. The nonlinear equations consisting of the average of (42) over each phase $r,(30)$ and (40), are solved for $\langle\boldsymbol{\alpha}\rangle_{r}, \widehat{\boldsymbol{\alpha}}^{(r)}$ and $\theta^{(r)}$ by a modification of the hybrid method of Powell (1970). In the present study use has been made of the MINPACK library (HYBRD1 routine).

At each step of this iterative procedure the residues of these equations are obtained by the following procedure :

2.1. The first and second moment of the strain are deduced from the relations (46). These moments depend on the thermoelastic constants (45).

2.2. The first and second moment per phase of the unknown field of internal variable $\boldsymbol{\alpha}$ at time $t_{n+1}$ are obtained by the relation (42) from the first and second moments of the strain field $\varepsilon$ computed at step 1 .

2.3. The residues are computed using the first moment of the field of internal variables $\boldsymbol{\alpha}$ computed at step 2.2 and the relations (30) and (40).

3. Once convergence is reached, the macroscopic stress $\boldsymbol{\Sigma}_{n+1}$ is obtained by taking the average of the microscopic stress field :

$$
\boldsymbol{\Sigma}_{n+1}=\left\langle\boldsymbol{\sigma}_{n+1}\right\rangle=\sum_{r=1}^{N} c^{(r)}\left[\mathcal{L}_{0}^{(r)}:\langle\boldsymbol{\varepsilon}\rangle_{r}+\boldsymbol{\rho}_{0}^{(r)}\right]
$$

Details about the convergence-rate of this algorithm are given in section 5.2.2.

The implementation of the TOE method is, in its principle, very similar to that of the secant method proposed in the first part of this study. The main differences lie first in the elastic moduli of the linear thermoelastic composite which are now anisotropic and second in the nonlinear equation for $\hat{\boldsymbol{\alpha}}^{(r)}$. Even the writing of this nonlinear equation requires the explicit knowledge of the 
third derivative of the dissipation potential which is not required in the secant method. Therefore, despite its similarities with the secant method, the TOE approach is more complex.

\subsection{The TOE procedure applied to composites with a single potential}

Although the TOE procedure was not initially meant for nonlinear composites governed by a single potential, it has also been applied in this case (Lahellec and Suquet, 2004, Rekik et al., 2005). For completeness, the main results of these authors are summarized here.

(1) The TOE procedure is exact to second-order in the contrast, as is the original SOE. Incidentally it provides an affine method which is exact to second-order in the contrast.

(2) In most cases which have been explored, the effective properties predicted by the TOE are close to the predictions of the SOE and exhibit similar trends as functions of the nonlinearity exponent or volume-fraction of the phases. Therefore it is likely that the limitations of the SOE, such as bounds violation in extreme cases, also apply to the TOE.

(3) In particular the field fluctuations in the phases do not influence the elastic moduli of the linear comparison composite, but affect the eigenstress.

(4) The SOE is known to exhibit a "duality gap" between the primal and dual formulations, in the sense that the predictions obtained by taking a Taylor expansion of the strain potential or of the stress potential are different. This duality gap is also present in the TOE.

(5) By construction, the TOE has no gap between the energy-based formulation and the affine (or stress-averaging) formulation. Therefore, in the TOE, the stress and strain fields in the linear comparison composite (thermoelastic) can be consistently taken as approximations of the actual stress and strain fields in the nonlinear composite. This is not the case in the SOE approach.

\section{Power-law materials}

In order to implement the TOE procedure, and in particular in order to solve the equation (30) for $\hat{\boldsymbol{\alpha}}^{(r)}$, one has to specify further the form of the potentials. Attention will be restricted here to isotropic nonlinear viscoelastic constituents with a power-law dissipation potential. The above theory applies also in principle to elasto-viscoplastic constituents (with a yield surface delimiting an elasticity domain), but we have not checked its practical implementation (we recall that it has been checked, in an unpublished study, that the secant 
method of part I works for elasto-viscoplastic materials as well).

\subsection{Detailed form of the operators}

The potentials are taken in the form :

$$
\left.\begin{array}{c}
w^{(r)}(\varepsilon, \boldsymbol{\alpha})=\frac{1}{2}(\varepsilon-\boldsymbol{\alpha}): \boldsymbol{L}^{(r)}:(\varepsilon-\boldsymbol{\alpha}), \boldsymbol{L}^{(r)}=3 k^{(r)} \boldsymbol{J}+2 \mu^{(r)} \boldsymbol{K}, \\
\varphi^{(r)}(\dot{\boldsymbol{\alpha}})=\frac{\sigma_{0}^{(r)} \dot{\varepsilon}_{0}}{m+1}\left(\frac{\dot{\alpha}_{\mathrm{eq}}}{\dot{\varepsilon}_{0}}\right)^{m+1} .
\end{array}\right\}
$$

The first two derivatives of $\varphi^{(r)}$ read as :

$$
\begin{gathered}
\frac{\partial \varphi^{(r)}}{\partial \dot{\boldsymbol{\alpha}}}\left(\dot{\boldsymbol{\alpha}}^{(r)}\right)=2 \mu_{v}^{(r)} \dot{\boldsymbol{\alpha}}^{(r)}, \\
\boldsymbol{L}_{v}^{(r)}=\frac{\partial^{2} \varphi^{(r)}}{\partial \dot{\boldsymbol{\alpha}}^{2}}\left(\dot{\boldsymbol{\alpha}}^{(r)}\right)=+\infty \boldsymbol{J}+2 \lambda_{v}^{(r)} \boldsymbol{E}^{(r)}+2 \mu_{v}^{(r)} \boldsymbol{F}^{(r)}=+\infty \boldsymbol{J}+2 \mu_{v}^{(r)}\left(m \boldsymbol{E}^{(r)}+\boldsymbol{F}^{(r)}\right),
\end{gathered}
$$

where $+\infty$ refers to the fact that $\varphi^{(r)}$ is finite only for incompressible $\dot{\boldsymbol{\alpha}}$, and :

$$
\mu_{v}^{(r)}=\frac{\sigma_{0}^{(r)}}{3 \dot{\varepsilon}_{0}}\left(\frac{\dot{\alpha}_{\mathrm{eq}}^{(r)}}{\dot{\varepsilon}_{0}}\right)^{m-1}, \quad \lambda_{v}^{(r)}=m \mu_{v}^{(r)}, \quad \boldsymbol{E}^{(r)}=\frac{2}{3} \frac{\dot{\boldsymbol{\alpha}}^{(r)} \otimes \dot{\boldsymbol{\alpha}}^{(r)}}{\left(\dot{\alpha}_{\mathrm{eq}}^{(r)}\right)^{2}}, \quad \boldsymbol{F}^{(r)}=\boldsymbol{K}-\boldsymbol{E}^{(r)} .
$$

Finally, the third derivative of $\varphi^{(r)}$ reads as :

$\boldsymbol{N}_{v}^{(r)}=\frac{4}{9}(m-1) \frac{\sigma_{0}^{(r)}}{\dot{\varepsilon}_{0}^{2}}\left(\frac{\dot{\alpha}_{\mathrm{eq}}^{(r)}}{\dot{\varepsilon}_{0}}\right)^{m-2}\left[\frac{3\left(\boldsymbol{K} \otimes \dot{\boldsymbol{\alpha}}^{(r)}\right)^{3 s}}{\dot{\alpha}_{\mathrm{eq}}^{(r)}}+\frac{2}{3}(m-3) \frac{\dot{\boldsymbol{\alpha}}^{(r)} \otimes \dot{\boldsymbol{\alpha}}^{(r)} \otimes \dot{\boldsymbol{\alpha}}^{(r)}}{\left(\dot{\alpha}_{\mathrm{eq}}^{(r)}\right)^{3}}\right]$

with the convention that $T_{i j k h m n}^{3 s}=\frac{1}{3}\left(T_{i j k h m n}+T_{k h m n i j}+T_{m n i j k h}\right)$.

\subsection{Determination of $\widehat{\boldsymbol{\alpha}}^{(r)}$.}

The equations (30) are solved for each phase separately and the unknowns $\widehat{\boldsymbol{\alpha}}^{(r)}$ can be decomposed into two terms respectively parallel and orthogonal to $\dot{\boldsymbol{\alpha}}^{(r)}$ :

$$
\widehat{\boldsymbol{\alpha}}^{(r)}=\beta \dot{\boldsymbol{\alpha}}^{(r)}+\dot{\boldsymbol{\alpha}}^{\perp(r)}, \quad \text { with } \quad \dot{\boldsymbol{\alpha}}^{\perp(r)}: \dot{\boldsymbol{\alpha}}^{(r)}=0 .
$$

Upon use of this decomposition and of the expression (53) of $\boldsymbol{N}$, the equation (30) reduces to : 


$$
\begin{aligned}
& \dot{\boldsymbol{\alpha}}^{\perp(r)}(\beta-1) \dot{\boldsymbol{\alpha}}_{\mathrm{eq}}^{(r)}+\frac{1}{3} \frac{\dot{\boldsymbol{\alpha}}^{(r)}}{\dot{\boldsymbol{\alpha}}_{\mathrm{eq}}^{(r)}}\left[\frac{3}{2}(\beta-1)^{2}\left(\dot{\boldsymbol{\alpha}}_{\mathrm{eq}}^{(r)}\right)^{2} m+\dot{\boldsymbol{\alpha}}^{\perp(r)}: \dot{\boldsymbol{\alpha}}^{\perp(r)}\right]= \\
& \frac{2 \boldsymbol{C}_{\dot{\alpha}}^{(r)}: \dot{\boldsymbol{\alpha}}^{(r)}}{\dot{\boldsymbol{\alpha}}_{\mathrm{eq}}^{(r)}}+\dot{\boldsymbol{\alpha}}^{(r)}\left[\frac{\boldsymbol{K}:: \boldsymbol{C}_{\dot{\alpha}}^{(r)}}{\dot{\boldsymbol{\alpha}}_{\mathrm{eq}}^{(r)}}+\frac{2}{3}(m-3) \frac{\boldsymbol{C}_{\dot{\alpha}}^{(r)}:: \dot{\boldsymbol{\alpha}}^{(r)} \otimes \dot{\boldsymbol{\alpha}}^{(r)}}{\left(\dot{\alpha}_{\mathrm{eq}}^{(r)}\right)^{3}}\right]
\end{aligned}
$$

The left-hand side of this equation has a component parallel to $\dot{\boldsymbol{\alpha}}^{\perp(r)}$ and another component parallel to $\dot{\boldsymbol{\alpha}}^{(r)}$. The right-hand side has a term parallel to $\dot{\boldsymbol{\alpha}}^{(r)}$ and another term parallel to $\boldsymbol{C}_{\dot{\alpha}}^{(r)}: \dot{\boldsymbol{\alpha}}^{(r)}$.

In all cases that we have investigated, due to symmetries in the microstructure and in the loading, the term $\boldsymbol{C}_{\dot{\alpha}}^{(r)}: \dot{\boldsymbol{\alpha}}^{(r)}$ has been observed numerically to be always parallel to $\dot{\boldsymbol{\alpha}}^{(r)}$. This was in particular the case for the two-dimensional periodic microstructures with two orthogonal planes of symmetry, subjected to a loading having the same axes of symmetry, such as the example studied in section 5.2.

Therefore, we will not discuss in general the resolution of equation (55) but, instead, work under the assumption that

$$
\boldsymbol{C}_{\dot{\alpha}}^{(r)}: \dot{\boldsymbol{\alpha}}^{(r)} \text { is parallel to } \dot{\boldsymbol{\alpha}}^{(r)} \text {. }
$$

Under this assumption, the solution $\widehat{\boldsymbol{\alpha}}^{(r)}$ of equation (55) satisfies

$$
\beta=1
$$

and

$$
\begin{aligned}
\dot{\boldsymbol{\alpha}}^{\perp(r)}: \dot{\boldsymbol{\alpha}}^{\perp(r)} & =3 \boldsymbol{K}:: \boldsymbol{C}_{\dot{\alpha}}^{(r)}+2(m-1) \frac{\boldsymbol{C}_{\dot{\alpha}}^{(r)}:: \dot{\boldsymbol{\alpha}}^{(r)} \otimes \dot{\boldsymbol{\alpha}}^{(r)}}{\left(\dot{\alpha}_{\mathrm{eq}}^{(r)}\right)^{2}} \\
& =3\left(m \boldsymbol{E}^{(r)}+\boldsymbol{F}^{(r)}\right):: \boldsymbol{C}_{\dot{\alpha}}^{(r)}=\frac{3}{2 \mu_{v}^{(r)}} \boldsymbol{L}_{v}^{(r)}:: \boldsymbol{C}_{\dot{\alpha}}^{(r)}
\end{aligned}
$$

The above equation does not allow for a complete determination of $\dot{\boldsymbol{\alpha}}^{\perp(r)}$ which is, in fact, not needed. The only expression involving $\dot{\boldsymbol{\alpha}}^{(r)}$ is the eigenstress $\boldsymbol{\tau}^{(r)}$ given by (33). In the case of power-law materials, and thanks to the result $\beta=1$, this expression reduces to :

$$
\boldsymbol{\tau}^{(r)}=2(m-1) \mu_{v}^{(r)}\left(-1+\frac{\dot{\boldsymbol{\alpha}}^{\perp(r)}: \dot{\boldsymbol{\alpha}}^{\perp(r)}}{9\left(\dot{\alpha}_{\mathrm{eq}}^{(r)}\right)^{2}}\right) \dot{\boldsymbol{\alpha}}^{(r)},
$$

where only (57) is required.

\subsection{The EIV approximation for power-law materials}

The EIV approximation introduces two unknowns $\boldsymbol{\alpha}_{n}^{(r)}$ and $\theta^{(r)}$ given by equations (41) and (40) respectively. The first relation (41) does not depend on the 
material under consideration (power-law or else) whereas the second relation (40) becomes for power-law materials, using the expression (51) for $\boldsymbol{L}_{v}^{(r)}$ :

$$
\theta^{(r)}=1-\sqrt{\frac{\left(m \boldsymbol{E}^{(r)}+\boldsymbol{F}^{(r)}\right)::\left\langle\left(\boldsymbol{\alpha}_{n}-\boldsymbol{\alpha}_{n}^{(r)}\right) \otimes\left(\boldsymbol{\alpha}_{n}-\boldsymbol{\alpha}_{n}^{(r)}\right)\right\rangle_{r}}{\left(m \boldsymbol{E}^{(r)}+\boldsymbol{F}^{(r)}\right)::\left\langle\left(\boldsymbol{\alpha}-\boldsymbol{\alpha}_{n}^{(r)}\right) \otimes\left(\boldsymbol{\alpha}-\boldsymbol{\alpha}_{n}^{(r)}\right)\right\rangle_{r}}}
$$

The sign in front of the square root, which was left undetermined in (40), has been taken to be a minus, for it was observed by Lahellec and Suquet (2006a) that the plus sign leads to predictions which are excessively stiff.

Note that the relations (57) and (59) require the evaluation of the contracted product (over four indices) of $\left\langle\left(\boldsymbol{\alpha}-\boldsymbol{\alpha}_{n}^{(r)}\right) \otimes\left(\boldsymbol{\alpha}-\boldsymbol{\alpha}_{n}^{(r)}\right)\right\rangle$ with the fourth-order tensor $m \boldsymbol{E}^{(r)}+\boldsymbol{F}^{(r)}$ which is itself proportional to $\boldsymbol{L}_{v}^{(r)}$ according to (51). It is clearly the case for (59). Regarding (57), we note that

$$
\begin{aligned}
& \boldsymbol{C}_{\dot{\alpha}}^{(r)}=\frac{1}{\Delta t^{2}}\left\langle\left(\boldsymbol{\alpha}-\boldsymbol{\alpha}_{n}\right) \otimes\left(\boldsymbol{\alpha}-\boldsymbol{\alpha}_{n}\right)\right\rangle_{r}-\langle\dot{\boldsymbol{\alpha}}\rangle_{r} \otimes\langle\dot{\boldsymbol{\alpha}}\rangle_{r} \\
& =\frac{\theta^{(r)}}{\Delta t^{2}}\left\langle\left(\boldsymbol{\alpha}-\boldsymbol{\alpha}_{n}^{(r)}\right) \otimes\left(\boldsymbol{\alpha}-\boldsymbol{\alpha}_{n}^{(r)}\right)\right\rangle_{r}-\langle\dot{\boldsymbol{\alpha}}\rangle_{r} \otimes\langle\dot{\boldsymbol{\alpha}}\rangle_{r}
\end{aligned}
$$

Therefore the product of $\boldsymbol{C}_{\dot{\alpha}}^{(r)}$ with $\boldsymbol{L}_{v}^{(r)}$, which is needed in (57), can be directly deduced from the product of $\left\langle\left(\boldsymbol{\alpha}-\boldsymbol{\alpha}_{n}^{(r)}\right) \otimes\left(\boldsymbol{\alpha}-\boldsymbol{\alpha}_{n}^{(r)}\right)\right\rangle_{r}$ with the fourth-order tensors $\boldsymbol{L}_{v}^{(r)}$ and from the product of $\langle\dot{\boldsymbol{\alpha}}\rangle_{r} \otimes\langle\dot{\boldsymbol{\alpha}}\rangle_{r}$ with the same tensor. This last product is straightforward, given the relation (51).

In conclusion, the equations to be solved involve only the first moment $\langle\boldsymbol{\alpha}\rangle_{r}$ in each phase of the field of internal variables $\boldsymbol{\alpha}$ and the projection of the second-order moment of this field on $\boldsymbol{L}_{v}^{(r)}$.

\subsection{The thermoelastic problem}

The projection of interest for $\boldsymbol{\alpha}$ is obtained from the same projection of the strain field $\varepsilon$ in the linear thermoelastic comparison composite with effective energy given by (44) with, for power-law materials :

$$
\mathcal{L}_{0}^{(r)}=3 k^{(r)} \boldsymbol{J}+2 \lambda_{0}^{(r)} \boldsymbol{E}^{(r)}+2 \mu_{0}^{(r)} \boldsymbol{F}^{(r)},
$$

with :

$$
\lambda_{0}^{(r)}=\frac{\mu^{(r)} \theta^{(r)} \lambda_{v}^{(r)} / \Delta t}{\mu^{(r)}+\theta^{(r)} \lambda_{v}^{(r)} / \Delta t} \text { and } \mu_{0}^{(r)}=\frac{\mu^{(r)} \theta^{(r)} \mu_{v}^{(r)} / \Delta t}{\mu^{(r)}+\theta^{(r)} \mu_{v}^{(r)} / \Delta t} .
$$


$k^{(r)}$ and $\mu^{(r)}$ refer to the linear elastic bulk and shear moduli of the phases. The eigenstress reads as :

$$
\begin{aligned}
& \boldsymbol{\rho}_{0}^{(r)}=\frac{\mu^{(r)}}{\mu^{(r)}+\theta^{(r)} \lambda_{v}^{(r)} / \Delta t}\left[2 \mu_{v}^{(r)}(1-m) \dot{\boldsymbol{\alpha}}^{(r)}\left(1-\frac{\dot{\boldsymbol{\alpha}}^{\perp(r)}: \dot{\boldsymbol{\alpha}}^{\perp(r)}}{9\left(\dot{\boldsymbol{\alpha}}_{\mathrm{eq}}^{(r)}\right)^{2}}\right)\right. \\
& \left.-\frac{\theta^{(r)} \lambda_{v}^{(r)} \boldsymbol{\alpha}_{n}^{(r)}}{\Delta t}\right] .
\end{aligned}
$$

The detailed expression of $f_{0}^{(r)}$ is not needed.

The first moment of $\boldsymbol{\varepsilon}$ is given by the relation (46 a) and its projections over $\boldsymbol{E}^{(r)}$ and $\boldsymbol{F}^{(r)}$ are obtained by derivation of the effective energy with respect to $\lambda_{0}^{(r)}$ and $\mu_{0}^{(r)}$ :

$$
\boldsymbol{E}^{(r)}::\langle\varepsilon \otimes \varepsilon\rangle_{r}=\frac{1}{c^{(r)}} \frac{\partial \widetilde{w}_{0}}{\partial \lambda_{0}^{(r)}} \text { and } \boldsymbol{F}^{(r)}::\langle\boldsymbol{\varepsilon} \otimes \varepsilon\rangle_{r}=\frac{1}{c^{(r)}} \frac{\partial \widetilde{w}_{0}}{\partial \mu_{0}^{(r)}} .
$$

\section{Examples}

\subsection{Orientation}

In this section, the accuracy of the TOE model derived in section 3 is assessed by comparing its predictions with the exact response (obtained numerically) of composite with specific microstructures. Comparisons with the secant procedure derived in the first part of this study also show the improvements brought by the present second-order procedure (TOE).

As in the first part of this study, it should be noted that there are two levels of approximation involved in the practical implementation of the present procedure. The first approximation (which is in fact two-fold) consists in the linearization procedure (TOE) and in the introduction of an effective internal variable (EIV). The second level of approximation pertains to the resolution of the thermoelastic problem. Our objective is to check the discrepancy introduced by the first set of approximations only. In order to minimize the error due to the resolution of the thermoelastic problem we have used either an exact (numerical) solution of the thermoelastic problem (section 5.2) or a class of microstructures for which an (almost) exact analytical solution of the thermoelastic problem is available (section 5.3).

The same five results are shown for each microstructure or material data.

(1) The first set of results, identified as "Exact (FEM)" or "Exact (FFT)", is obtained by solving numerically the nonlinear nonlinear viscoelastic 
problem, either by the Finite Element Method (FEM) for the simple periodic microstructure of section 5.2 , or by a method based on Fast Fourier Transforms for the cylinder assemblage in section 5.3.

(2) The second set of results, identified as "TOE (FEM)", is obtained by approximating the nonlinear incremental potential (4) by the quadratic potential $J_{\text {toe }}^{(r)}$ given by the relation (32). The corresponding thermoelasticity problem (34) involves nonuniform eigenstrain fields and is solved by the FEM.

(3) The third set of results, identified as "TOE (EIV+HS)", is obtained by approximating, in the linear incremental potential, the nonuniform fields of internal variables by piecewise uniform "effective internal variables", leading to the thermoelastic problem (44). In addition, the effective energy of this thermoelastic problem is evaluated either by one of the classical Hashin-Shtrikman bounds (in the case of the composite cylinder assemblage) or by a generalized Hashin-Shtrikman bound due to Suquet (1990) (see also Lahellec and Suquet, 2006a) in the case of the periodic microstructure of section 5.2. In all cases, the Hashin-Shtrikman bounds are obtained with the matrix as the reference medium.

(4) The fourth set of results, identified as "SEC (FEM)" refers to the intermediate secant model (secant linearization without the further approximation of effective internal variables). More details about this model were given in the first part of this study.

(5) The fifth set of results, identified as "SEC (EIV+HS)", corresponds to the complete secant model of part I of this paper. The Hashin-Shtrikman bounds are obtained following the same lines as in the third set of results.

The focus will be on two-phase fiber composites. The fibers, aligned with the direction of $\boldsymbol{e}_{3}$, will be identified as phase 1, whereas the continuous phase, called the matrix, will be identified as phase 2 . Two microstructures will be considered, a simple periodic microstructure consisting of a circular fiber in a square unit-cell repeated periodically (figure 6a) and the assemblage of composite cylinders already used in part I (Lahellec and Suquet, 2006b) of this study. The composites are subjected to an imposed macroscopic shear strain :

$$
\boldsymbol{E}(t)=E_{11}(t)\left(\boldsymbol{e}_{1} \otimes \boldsymbol{e}_{1}-\boldsymbol{e}_{2} \otimes \boldsymbol{e}_{2}\right)
$$

The strain $E_{11}(t)$ is increased monotonically with a constant strain-rate.

\subsection{Square array of circular fibers}

\subsubsection{Material data}

We consider here an elastically-reinforced matrix with a fiber volume-fraction ranging from $c^{(1)}=0.05$ to $c^{(1)}=0.5$. The fibers are isotropic and linearly 
elastic whereas the matrix is nonlinear viscoelastic with potentials in the form (50). The material data are :

- Fibers : $E=400 \mathrm{GPa}, \nu=0.2$.

- Matrix : $E=70 \mathrm{GPa}, \nu=0.3, \sigma_{0}=480 \mathrm{MPa}, \dot{\varepsilon}_{0}=10^{-2} \mathrm{~S}^{-1}$.

In addition, the matrix rate-sensitivity exponent $m$ varies from 0.1 to 1 . The loading is given by (63) with a constant strain-rate $\dot{E}_{11}=510^{-3} \mathrm{~s}^{-1}$.

\subsubsection{Implementation of the TOE approach}

The TOE approach has been implemented along the lines of section 3.3. In the present case of purely elastic fibers (phase 1), the unknowns of the problem are $\langle\boldsymbol{\alpha}\rangle_{2}, \widehat{\boldsymbol{\alpha}}^{(2)}$ and $\theta^{(2)}$. In all examples investigated in the present study, the iterative algorithm of section 3.3 has always converged to a solution. We believe that this solution is unique (at least in rate-dependent problems) but we have no proof of this assertion. Convergence is reached when the relative errors between two iterates for $\theta^{(2)}, \widehat{\boldsymbol{\alpha}}^{(2)}$ and $\langle\boldsymbol{\alpha}\rangle_{2}$ is less than a given threshold (typically $10^{-8}$ ). With this criterion and for a typical example of the present section corresponding to $m=0.2$ and $c^{(1)}=0.3$, convergence was attained in 20 to 40 iterations (typically), the slowest convergence being observed in the transient regime where elastic and viscous effects are of the same order of magnitude. For the same problem, the secant method of part I took between 15 and 30 iterations to converge.

\subsubsection{Results and discussion}

As already noted in the first part of this study, the main trends of the stressstrain curve of these composites under monotone loading at constant strainrate are typically an initial linear-elastic response for small strains, then a transient part which finally leads to a plateau corresponding to the purely viscous response of the composite. This plateau is characterized by a stress $\Sigma_{11}^{\infty}$. These trends are shown in figure 3 where the five sets of results described in section 5.1 are plotted for different fiber volume-fraction or rate-sensitivity exponents. Figures 3 (a), (b) and (c) correspond to the same exponent $m=0.2$ and to different volume fractions $c^{(1)}=0.2,0.3$ and 0.4 . Figures 3 (d), (e) and (f) correspond to the same fiber volume-fraction $c^{(1)}=0.4$ with different rate-sensitivity exponents $m=0.1,0.5$ et 1 .

The following comments can be made :

(1) The error introduced by the EIV approximation and by the HS bound is measured for both models, SEC and TOE, by the distance between $\mathrm{SEC}(\mathrm{FEM})$ and SEC(EIV+HS) (same for TOE). As can be seen this error 


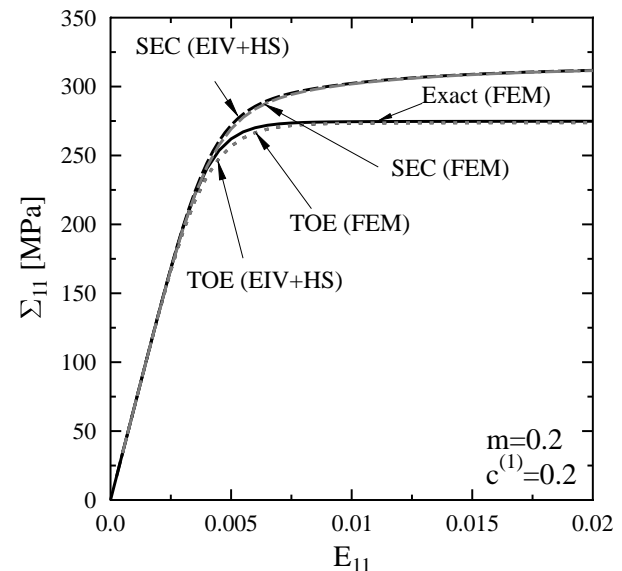

(a)

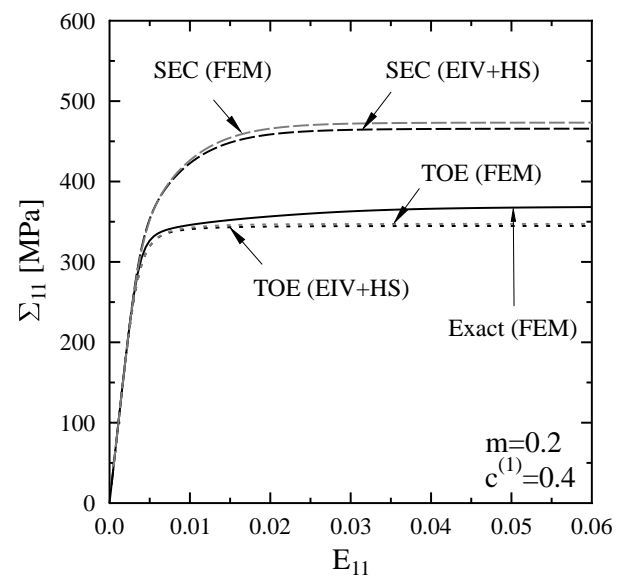

(c)

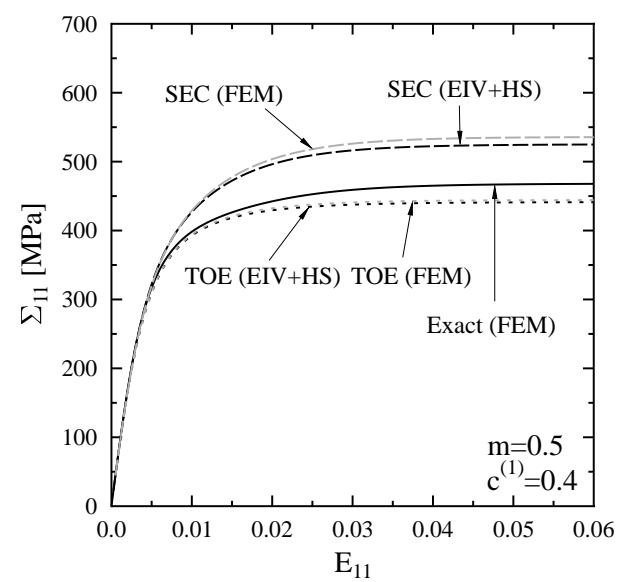

(e)

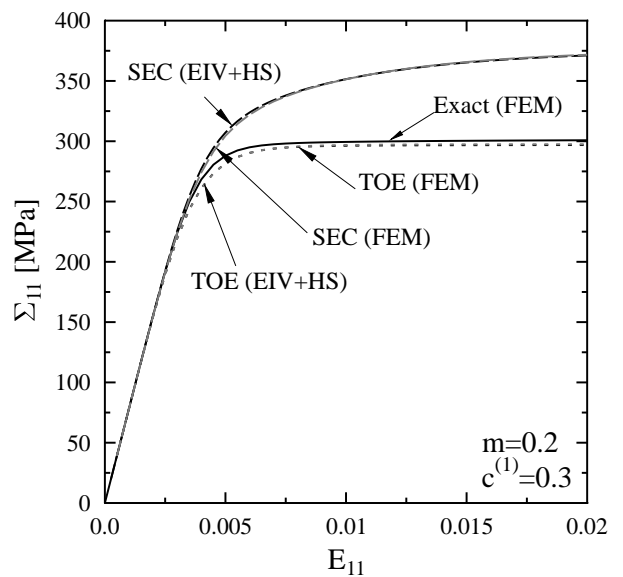

(b)

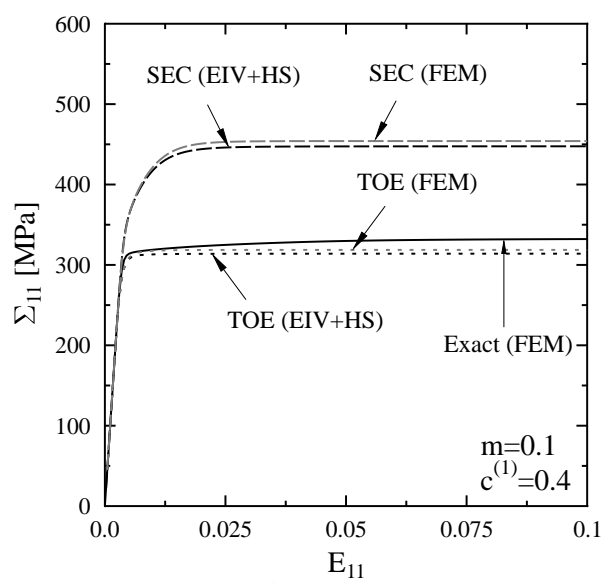

(d)

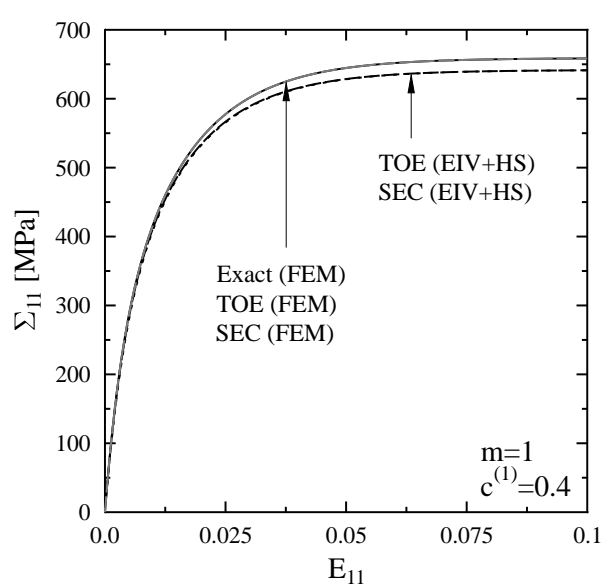

(f)

Fig. 3. Square array of circular fibers. Elastic fibers in a nonlinear viscoelastic matrix. Response of the composite under in-plane shear for different fiber volume-fractions and for different rate-sensitivity exponents. $(a): c^{(1)}=0.2$ and $m=0.2 .(b): c^{(1)}=0.3$ and $m=0.2 .(c): c^{(1)}=0.4$ and $m=0.2 .(d): c^{(1)}=0.4$ and $m=0.1$. $(e): c^{(1)}=0.4$ and $m=0.5$. $(f): c^{(1)}=0.4$ and $m=1$. 
is small and depends on the size of the transient part of the response.

(2) Except in the linear case where no linearization is needed, the secant model overestimates the stiffness of the composite. Although it has not been shown to be a rigorous upper bound, it tends to lie systematically above the exact results. The discrepancy increases with the fiber volumefraction and with the nonlinearity (the smaller $m$, the larger the nonlinearity).

(3) By contrast with the SEC model, the TOE model underestimates the composite stiffness. The discrepancy, which is in all cases less than in the SEC model, increases with the fiber volume-fraction. However, there is a specific fiber volume-fraction around $c^{(1)}=0.4$ where it seems that the nonlinearity does not affect the error.

(4) In all cases, the largest error is observed in the asymptotic regime. Therefore the error introduced by the model can be measured by comparing the asymptotic stresses $\Sigma_{11}^{\infty}$. This is done in Figure 4 as a function of the fiber volume-fraction for $m=1$ and $m=0.2$.

(5) Comparison between the strain fields in the unit-cell can be performed from the full-field computations, either in the actual nonlinear composite or in the thermoelastic comparison composites introduced in the SEC and TOE procedures. The corresponding snapshots of the $\varepsilon_{11}$ component of the strain are shown in figure 6, corresponding to the exact result (figure $6 \mathrm{~b}$ ), the prediction of SEC(FEM) (figure 6c) and the prediction of TOE(FEM) (figure 6d). White or light grey correspond to high deformations, whereas black corresponds to low deformation. The field estimated by the TOE model is in good agreement with the actual strain field. The better agreement of the strain fields compensates a higher complexity of the TOE model as compared to the SEC model.

\subsection{Composite cylinder assemblage}

The microstructures considered in the present section have been described in the first part of this study and in Moulinec and Suquet (2003) and Idiart et al. (2006b). They approach, as closely as possible within computational limitations, an ideal composite cylinder assemblage for which one of the HashinShtrikman bounds is a sharp estimate for isotropic phases. Two fiber volumefractions have been considered : $c^{(1)}=0.21$ and $c^{(1)}=0.41$. Both phases are nonlinear viscoelastic with potentials in the form (50) with the same ratesensitivity exponent $m$. The material data were :

- Fibers : $E=100 \mathrm{GPa}, \nu=0.45, \dot{\varepsilon}_{0}=1 \mathrm{~s}^{-1}$.

- Matrix : $E=100 \mathrm{GPa}, \nu=0.45, \sigma_{0}=1 \mathrm{GPa}, \dot{\varepsilon}_{0}=1 \mathrm{~s}^{-1}$. 


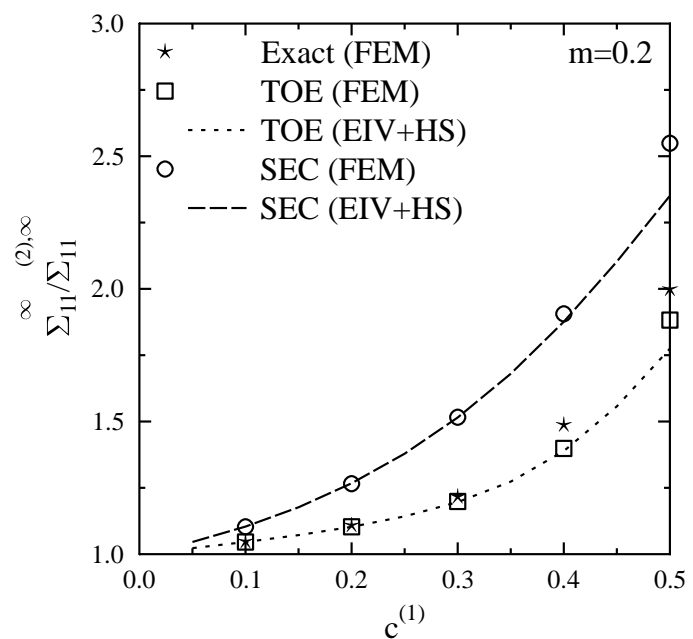

(a)

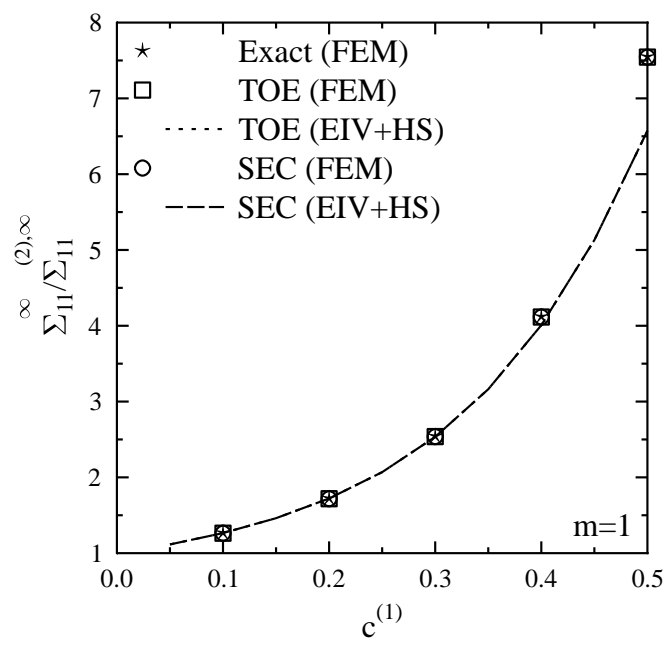

(b)

Fig. 4. Square array of circular fibers. Elastic fibers in a nonlinear viscoelastic matrix. In-plane macroscopic shear. Asymptotic macroscopic stress $\Sigma_{11}^{\infty}$ as a function of the fiber volume-fraction $c^{(1)}$ for two different rate-sensitivity exponents. a) $m=0.2$, b) $m=1$. The asymptotic stress is normalized by the stress in the unreinforced matrix under the same loading conditions, $\Sigma_{11}^{(2), \infty}$.

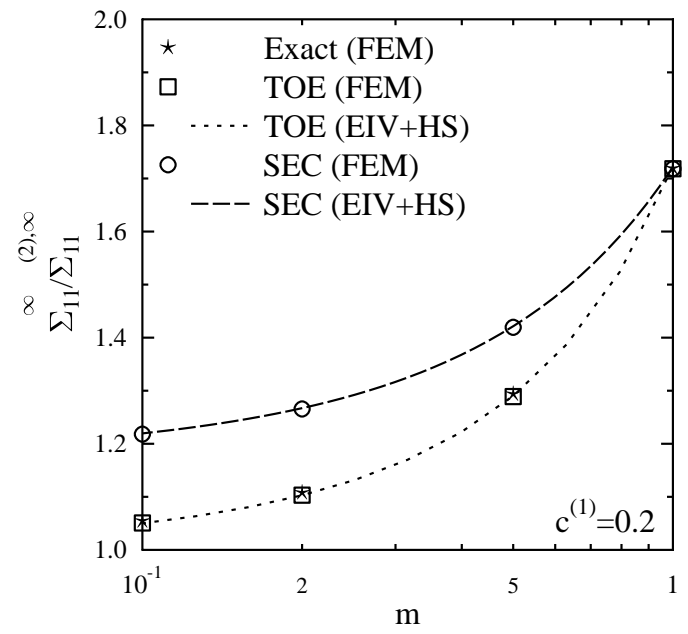

(a)

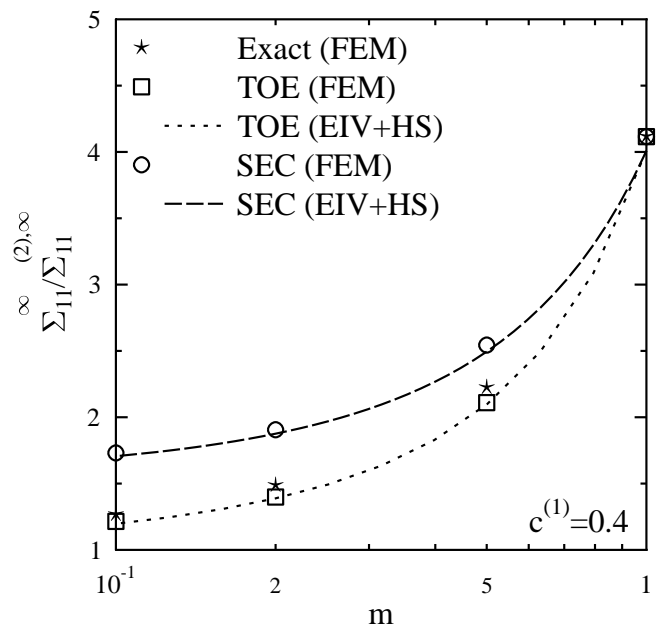

(b)

Fig. 5. Square array of circular fibers. Elastic fibers in a nonlinear viscoelastic matrix. In-plane macroscopic shear. Asymptotic macroscopic stress $\Sigma_{11}^{\infty}$ as a function of the rate-sensitivity exponent $m$ of the matrix for two different volume fractions. a) $c^{(1)}=0.2$, b) $c^{(1)}=0.4$. The asymptotic stress is normalized by the stress in the unreinforced matrix under the same loading conditions, $\Sigma_{11}^{(2), \infty}$. 


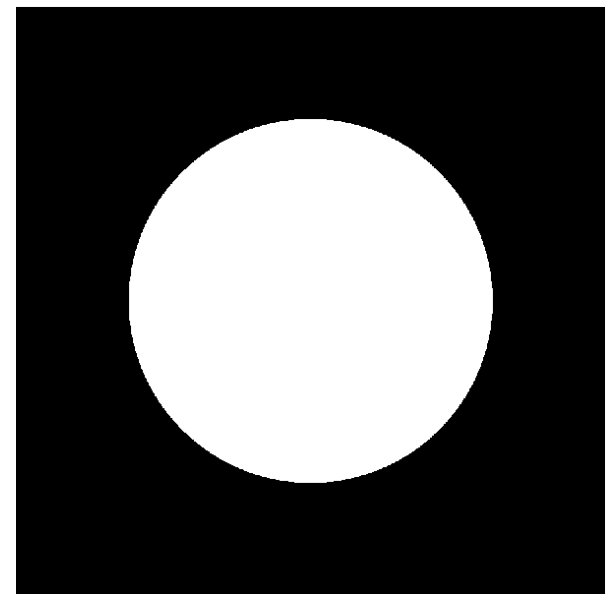

(a)

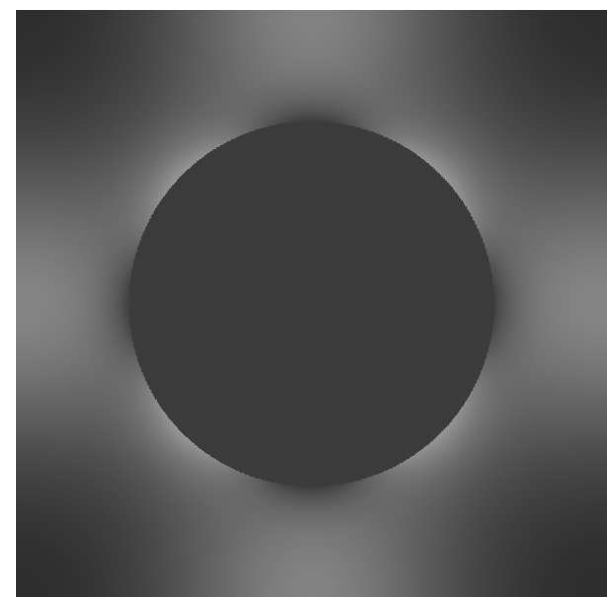

(c)

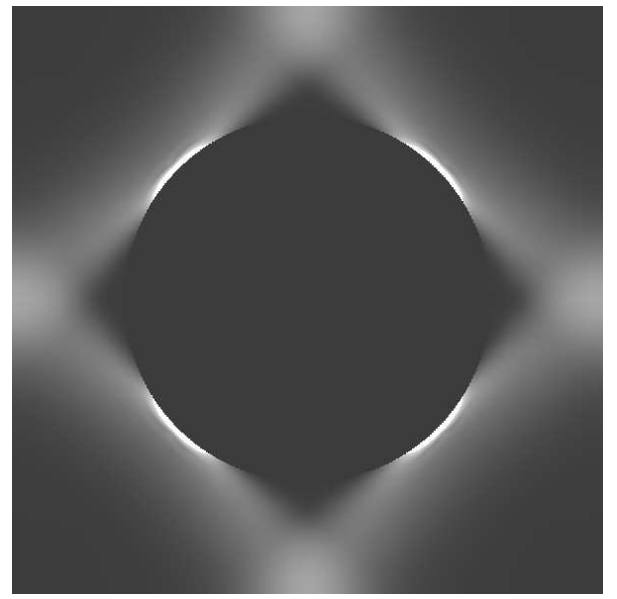

(b)

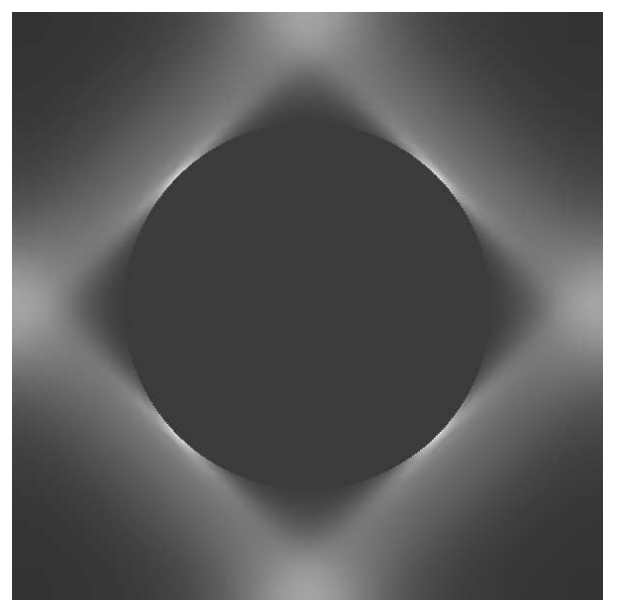

(d)

Fig. 6. Square array of circular fibers. Elastic fibers in a nonlinear viscoelastic matrix $(m=0.1)$. In-plane macroscopic shear (63) . (a): unit-cell $\left(c^{(1)}=0.3\right)$. (b), (c) and (d) snapshots of the component $\varepsilon_{11}$ of the strain field and, (b) Exact FEM calculations, (c) SEC(FEM), (d) TOE(FEM).

Two different cases for the flow-stress in the fibers were considered, $\sigma_{0}^{(1)}=$ 5 and $\sigma_{0}^{(1)}=0.2 \mathrm{GPa}$, corresponding to fiber-reinforced or fiber-weakened composites. The rate-sensitivity exponent $m$, identical for both phases, was varied from 0.1 to 1 . The applied loading was a pure shear (63) with a constant strain-rate $\dot{E}_{11}=510^{-1} \mathrm{~s}^{-1}$.

Three predictions for the overall stress-strain response of the composites are presented in figure 7 in the fiber-reinforced case and in figure 8 for the fiberweakened case. The exact result corresponds to the full-field computation performed by FFT. The TOE(EIV+HS) prediction corresponds to the TOE linearization together with the EIV approximation. In addition the thermoelas- 


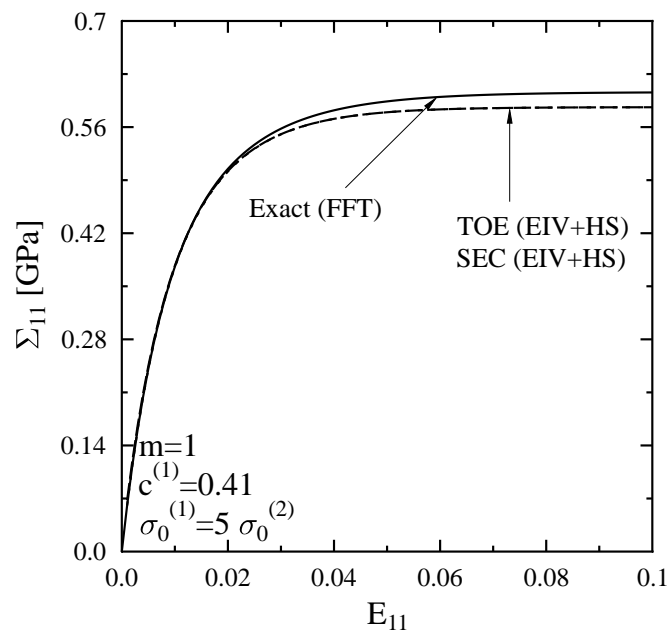

(a)

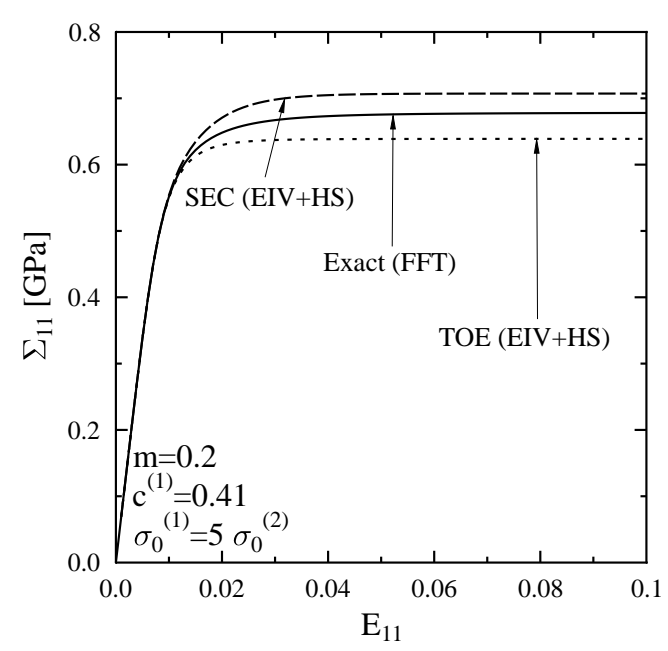

(b)

Fig. 7. Composite cylinder assemblage. Fiber-reinforced case $\sigma_{0}^{(1)} / \sigma_{0}^{(2)}=5$. (a): $c^{(1)}=0.41$ and $m=1 .(b): c^{(1)}=0.41$ and $m=0.2$.

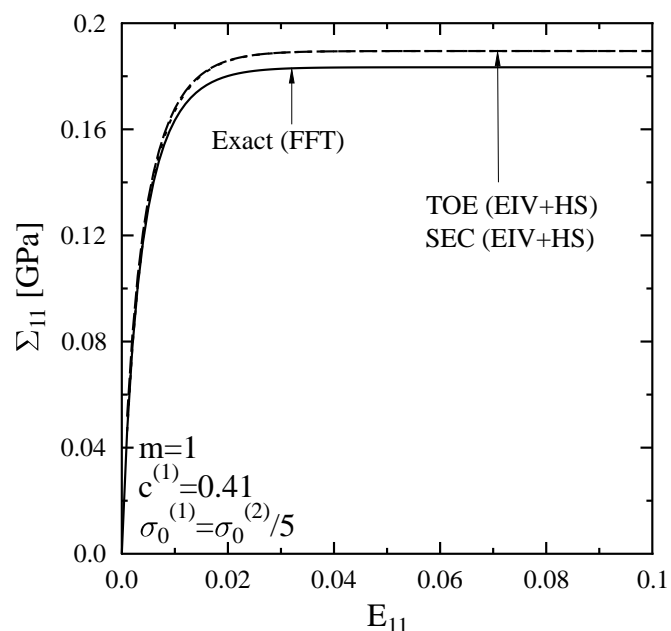

(a)

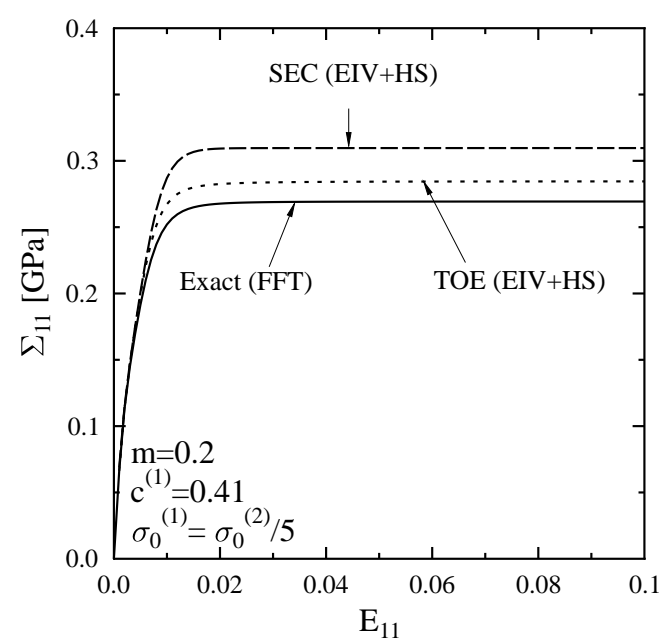

(b)

Fig. 8. Composite cylinder assemblage. Fiber-weakened case $\sigma_{0}^{(1)} / \sigma_{0}^{(2)}=0.2$. (a): $c^{(1)}=0.41$ and $m=1$. (b): $c^{(1)}=0.41$ and $m=0.2$.

tic is solved using the relevant Hashin-Shtrikman bound for two-dimensional composites (lower bound in the fiber-reinforced case, upper bound in the fiberweakened case).

When $m=1$ (linear viscoelasticity), the secant and second-order predictions coincide, since there is no linearization needed. In the fiber-reinforced case, the predictions are softer than the exact result because of the use of the lower HS bound (conversely in the fiber-weakened case, the model is stiffer than the exact results). These bounds are accurate rigorous bounds for the 
microstructure under consideration, but not exact results.

When $m<1$, the fiber-reinforced case confirms the trends of the preceeding section: for high enough nonlinearity, the SEC predictions are stiffer than the exact results whereas the TOE predictions tend to lie on the compliant side. In the fiber-weakened case, the SEC prediction remains too stiff whereas the TOE prediction lies now on the stiff side, but with a better agreement than the SEC model.

In all cases the error is maximal in the asymptotic regime and the different models can be compared by inspecting the asymptotic stresses $\Sigma_{11}^{\infty}$. This is done in figure 9 where the three predictions for the asymptotic stress are compared when the rate-sensitivity exponent $m$ is varied. The overall stress has been normalized by the similar asymptotic stress in the matrix alone under the same loading conditions. The TOE model improves on the SEC model in all cases. The improvement is especially significant for the fiber-weakened composite where the trends of the SEC model are opposite to the trends of the exact results (as $m$ varies) whereas the TOE model captures well the influence of $m$.

\section{Closing remarks}

This study is devoted to nonlinear composite materials comprised of nonlinear viscoelastic constituents whose constitutive relations can be derived from two thermodynamic potentials. The present study is based on an incremental variational principle derived in the first part of this paper (Lahellec and Suquet, 2006b). In the present paper the application of a new second-order procedure, where a modification of the initial second-order of Ponte Castañeda (1996) has been proposed to close the gap between the so-called affine and energy based approach, has been investigated.

Our main findings can be summarized as follows :

(1) The prediction obtained by a procedure following strictly the original second-order method showed inconsistent features due to the difference between the stress obtained by derivation of the energy and the average stress in the linear thermoelastic composite (gap between the affine and strain-energy based approaches).

(2) By adding to the second order expansion of the dissipation potential a term depending on the third derivative of the potential to the eigenstress, it has been possible to close this gap and to recover consistent results. The resulting scheme remains exact to second-order in the contrast of the phases. 


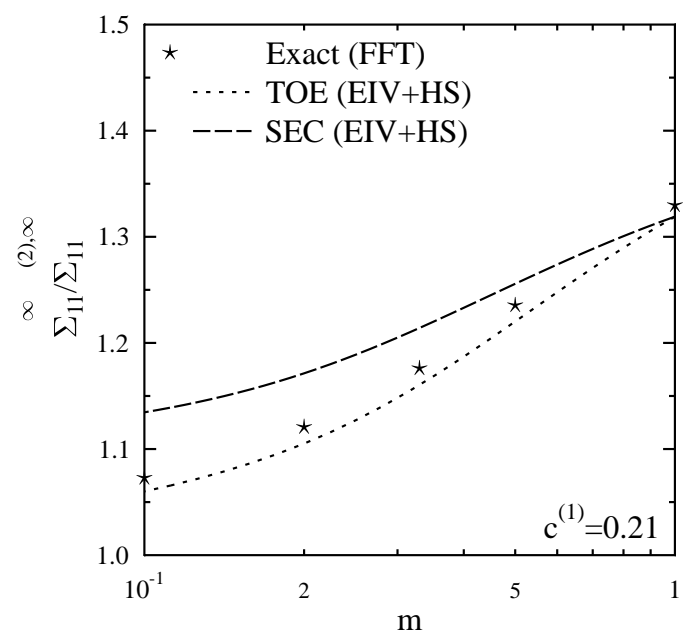

(a)

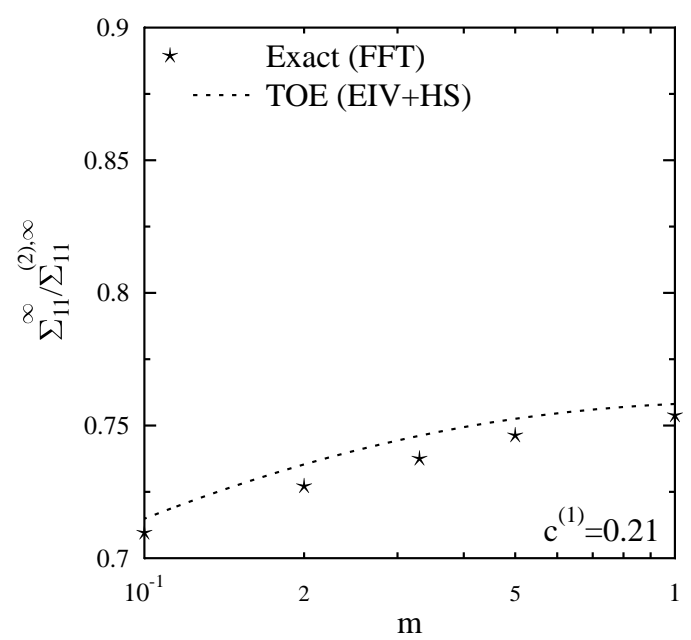

(c)

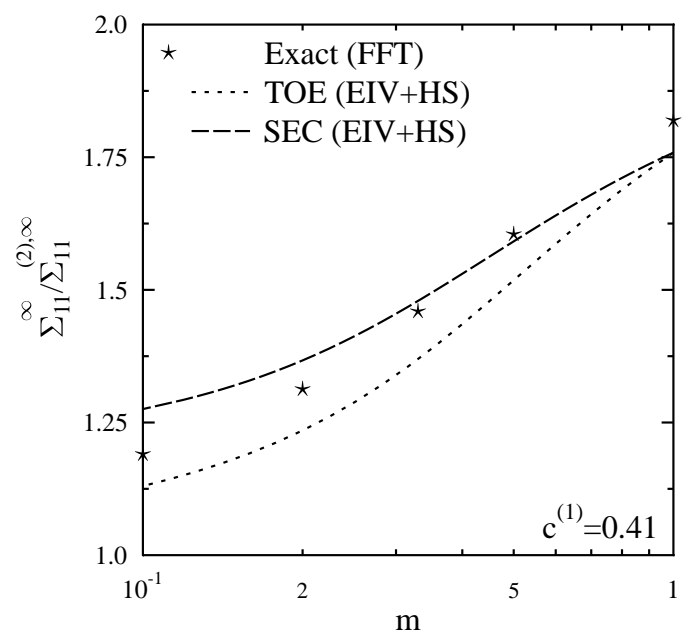

(b)

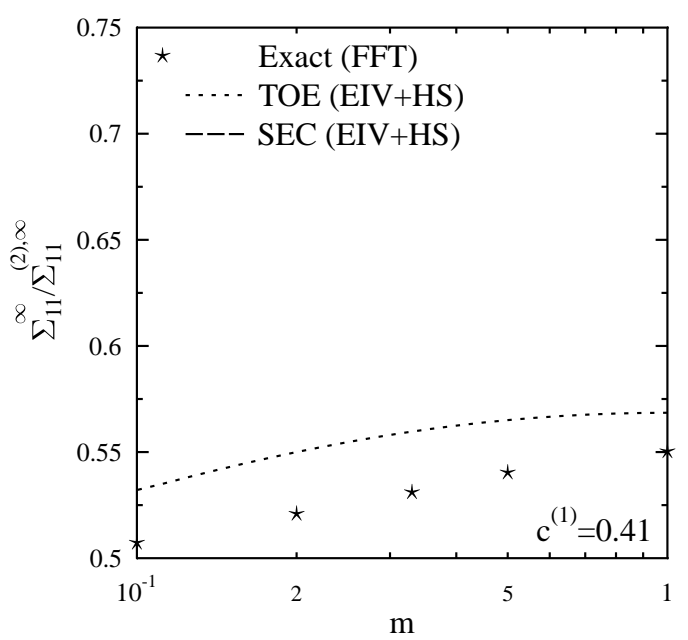

(d)

Fig. 9. Composite cylinder assemblage. Asymptotic macroscopic stress $\Sigma_{11}^{\infty}$ as a function of the nonlinearity exponent $m$. The asymptotic stress is normalized by the stress in the unreinforced matrix under the same loading conditions, $\Sigma_{11}^{(2), \infty}$. Comparison between the exact result "Exact (FEM)", the linearization scheme solved with the EIV approximation associated with the Hashin-Shtrikman method "TOE $(E I V+H S)$ " and "SEC $(E I V+H S)$ ". (a) $: \sigma_{0}^{(1)} / \sigma_{0}^{(2)}=5$ and $c^{(1)}=0.21$, (b) $: \sigma_{0}^{(1)} / \sigma_{0}^{(2)}=5$ and $c^{(1)}=0.41,(c): \sigma_{0}^{(1)} / \sigma_{0}^{(2)}=0.2$ and $c^{(1)}=0.21,(d)$ : $\sigma_{0}^{(1)} / \sigma_{0}^{(2)}=0.2$ and $c^{(1)}=0.41$. 
(3) In addition to this more consistent linearization, an "effective internal variable approach" has been proposed in order to reduce the problem to that for a $N$-phase thermoelastic composite. Examples shown in section section 5 show that this approximation is indeed very accurate.

(4) Comparison with the secant model (SEC) proposed in the first part of this study (Lahellec and Suquet, 2006b) shows on one hand that the predictions of this second-order procedure (TOE) are more accurate than those obtained with the SEC model. On the other hand, they are obtained at the expense of a more complicated implementation, making use of an anisotropic thermoelastic composite and also relying on assumptions which hold true in the two-dimensional examples that have been investigated here but which remain to be checked in more general (3-dimensional) analyses.

There remains room for significant improvement of the proposed schemes.

(1) First the modification of the SOE proposed by Lahellec and Suquet (2004) is not optimal. Although it achieves its initial goal, which was to close the gap between the overall stress evaluated either from the energy formulation or from the average stress in the linear thermoelastic composite, its predictions remain close to that of the original SOE. As such, its limitations are similar: there remains a significant gap between the primal and the dual formulations and its predictions can be inaccurate in extreme cases. A first improvement could be brought by using a more refined second-order procedure such as those recently developed by Idiart et al. (2006a).

(2) Second the procedure used to derive the "effective internal variable" is directly inspired from the incompressible isotropic case of the first part of this study. It should be improved by considering directly the EIV in the thermoelastic potential, instead of treating this step separately.

\section{Acknowledgements}

This study is part of program supported by the Centre National de la Recherche Scientifique under grant CNRS/NSF n 14555 .

\section{References}

Brenner, R., Masson, R., 2005. Improved affine estimates for nonlinear viscoelastic composites. Eur. J. Mechanics A/Solids 24, 1002-1015.

Germain, P., Nguyen, Q., Suquet, P., 1983. Continuum Thermodynamics. J. Appl. Mech. 50, 1010-1020. 
Idiart, M., Danas, K., Ponte Castañeda, P., 2006a. Second-order theory for nonlinear composites and application to isotropic constituents. C. R. Mécanique 334, 575-581.

Idiart, M., Moulinec, H., Ponte Castañeda, P., Suquet, P., 2006b. Macroscopic behavior and field fluctuations in viscoplastic composites: second-order estimates versus full-field simulations. J. Mech. Phys. Solids 54 , 1029-1063.

Lahellec, N., Suquet, P., 2004. Nonlinear composites: a linearization procedure, exact to second-order in contrast and for which the strain-energy and the affine formulations coincide. C. R. Mécanique 332, 693-700.

Lahellec, N., Suquet, P., 2006a. Effective behavior of linear viscoelastic composites: a time-integration approach. Int. J. Solids Structures 44, 507-529.

Lahellec, N., Suquet, P., 2006b. On the effective behavior of nonlinear inelastic composites: I incremental variational principles. Submitted.

Masson, R., Bornert, M., Suquet, P., A., Zaoui., 2000. An affine formulation for the prediction of the effective properties of nonlinear composites and polycrystals. J. Mech. Phys. Solids 48, 1203-1227.

Masson, R., Zaoui, A., 1999. Self-consistent estimates for the rate-dependent elastoplastic behaviour of polycrystalline materials. J. Mech. Phys. Solids 47, 1543-1568.

Mialon, P., 1986. Eléments d'analyse et de résolution numérique des relations de l'élasto-plasticité. EDF Bulletin de la Direction des Etudes et recherches. Série C. Mathématiques, Informatique, Nº3, 57-89.

Miehe, C., 2002. Strain-driven homogenization of inelastic micro-structures and composites based on an incremental variational formulation. Int. J. Numer. Meth. Engng 55, 1285-1322.

Moulinec, H., Suquet, P., 2003. Intraphase strain heterogeneity in nonlinear composites: a computational approach. Eur. J. Mech.: A/ Solids 22, 751770 .

Ortiz, M., Stainier, L., 1999. The variational formulation of viscoplastic constitutive updates. Comput. Methods Appl. Mech. Engrg 171, 419-444.

Ponte Castañeda, P., 1996. Exact second-order estimates for the effective mechanical properties of nonlinear composite materials. J. Mech. Phys. Solids 44, 827-862.

Ponte Castañeda, P., 2002. Second-order homogenization estimates for nonlinear composites incorporating field fluctuations. I - Theory. J. Mech. Phys. Solids 50, 737-757.

Ponte Castañeda, P., Suquet, P., 1998. Nonlinear composites. Adv. Appl. Mech. 34, 171-302.

Powell, M.J.D., 1970. A hybrid method for nonlinear algebraic equation. In: P Rabinowitz Eds, Numerical Methods for Nonlinear Algebraic Equations, Gordon and Breach Pub.

Rekik, A., Bornert, M., Auslender, F., Zaoui, A., 2005. A methodology for an accurate evaluation of the linearization procedures in nonlinear mean field homogenization. C. R. Mécanique 333, 789-795.

Suquet, P., 1990. Une méthode simplifiée pour le calcul des propriétés 
élastiques de matériaux hétérogènes à structure périodique. C. R. Acad. Sci. Paris série IIb 311, 769-774.

Suquet, P., Ponte Castañeda, P., 1993. Small-contrast perturbation expansions for the effective properties of nonlinear composites. C.R. Acad. Sc. Paris II $317,1515-1522$. 